\title{
Der Konjunktiv als Mittel der Bedeutungsschattierung
}

\author{
[The subjunctive as a means to modify meaning] \\ http://dx.doi.org/10.11606/1982-8837192953
}

\section{Veronika Geith ${ }^{1}$}

\begin{abstract}
The correct use of subjunctive can be difficult for learners of German. To understand these difficulties, it is important to recognize the specificities of the German subjunctive. Therefore, this article begins with pointing out the differences between the subjunctive and the two other German moods, indicative and imperative. It continues with a description of particularities of the subjunctive as a means of modality. Subsequently, the roles that can be taken by subjunctive I and II are presented. The article concludes with a discussion of how to teach the German subjunctive in the classroom.
\end{abstract}

Keywords: Modality in German; meaning and function of subjunctive I and II; German as a foreign language.

Resumo: Para quem estuda alemão, o uso do subjuntivo constitui um desafio. Para poder compreender isso é preciso conhecer as particularidades do subjuntivo em alemão. Por isso, esse artigo esclarece primeiramente as diferenças entre o modo subjuntivo e os dois outros modos alemães: o indicativo e o imperativo. O artigo mostra a seguir as particularidades do subjuntivo, sendo um meio da modalidade. Subsequente, apresentam-se as funções que podem ser cumpridas pelo subjuntivo I e subjuntivo II. O artigo conclui, assim, com uma discussão sobre o ensino do subjuntivo alemão na sala de aula com língua estrangeira.

Palavras-chave: Modalidade na língua alemã; importância e funções do subjuntivo I e II; alemão como língua estrangeira.

Zusammenfassung: Die richtige Verwendung des Konjunktivs bereitet vielen Deutschlernern/innen Schwierigkeiten. Um diese Schwierigkeiten nachvollziehen zu können, ist es wichtig, die Besonderheiten des deutschen Konjunktivs zu kennen. Darum wird in diesem Aufsatz zuerst der Modus Konjunktiv von den anderen beiden Modi des Deutschen, dem Indikativ und dem Imperativ, abgegrenzt, und herausgestellt, worin sich die Modi des Deutschen unterscheiden. Anschließend werden Besonderheiten des Konjunktivs als einem Mittel der Modalität dargestellt. Sodann wird im Einzelnen dargestellt, welche Funktionen Konjunktiv I und II übernehmen können. Der Aufsatz schließt mit einer Diskussion über das Unterrichten des Konjunktivs im DaF-Unterricht.

Stichwörter: Modalität im Deutschen; Bedeutung und Funktion von Konjunktiv I und II; Deutsch als Fremdsprache.

\footnotetext{
${ }^{1}$ Universidade Federal de Pelotas, Centro de Letras e Comunicação, Rua Gomes Carneiro, 1, 96010-610, Pelotas, RS, Brasil. E-mail: veronika@geith-weine.de
} 


\section{Einleituns}

Das Deutsche besitzt drei Modi: Indikativ, Konjunktiv und Imperativ. Viele andere Sprachen verfügen darüber hinaus auch noch über weitere Modi. Das Französische hat das Konditional zum Ausdruck einer möglichen Wirklichkeit; das Griechische, Türkische und Finnische verfügen über den Optativ zur Darstellung erfüllbarer Wünsche; im Türkischen kann der Suppositiv zum Anstellen von Vermutungen verwendet werden; und das Arabische besitzt den Energikus, um etwas nachdrücklich zu behaupten (vgl. BUßMANN 2002: 444).

Der Konjunktiv ist eine Verbkategorie, die in allen indoeuropäischen Sprachen und in vielen nichtindoeuropäischen Sprachen vorhanden ist (vgl. GLÜCK 2000: 365). Aber da das Deutsche nur über drei Modi verfügt, kann es sein, dass der Konjunktiv eine grammatische oder pragmatische Funktion übernimmt, die in der Ausgangssprache der Deutschlerner/innen von einem anderen bzw. einem eigenen Modus kodiert wird. Hieraus können sich Schwierigkeiten für Lerner/innen ergeben. Hat die eigene Muttersprache ein anderes Modussystem oder kennt sie den Modus Konjunktiv nicht, so muss man sich als Deutschlerner/in erst mit den Funktionen und der Bedeutung des Konjunktivs vertraut machen. Diese Bedeutungen sind aber schwer auf einen Nenner zu bringen (vgl. Jung 1984: 223). Daher kann man auch verstehen, warum der deutsche Konjunktiv eine Lernschwierigkeit für Nicht-Muttersprachler/innen darstellt. Wie eine Studie zeigt, sind sich Lerner/innen unsicher hinsichtlich dessen, was Konjunktiv I und Konjunktiv II leisten können (vgl. GöTZE et al. 1978: 27).

Darüber hinaus ergeben sich Schwierigkeiten bei der Anwendung dieses Modus (vgl. ebd.: 27). Denn hat man als Deutschlerner/in theoretisch die Verwendungsweise des Konjunktivs verstanden, so kann es gut sein, dass man von der tatsächlichen Gebrauchsweise verwirrt wird. Im Fremdsprachenunterricht wird zwar gelehrt, dass der Konjunktiv II immer dann den Konjunktiv I ersetzt, wenn dieser nicht eindeutig ist. Aber diese Regel wird nicht immer strikt befolgt: teilweise bleiben nicht eindeutige Konjunktiv-I-Formen bestehen, teilweise werden Konjunktiv-II-Formen auch dann verwendet, wenn der Konjunktiv-I durchaus eindeutig wäre (vgl. ENGEL 1996: 423). Aufgrund dessen kann man verstehen, dass der Konjunktiv eine Lernschwierigkeit für Nichtmuttersprachler/innen darstellt und dass hier erhöhter Übungsbedarf besteht. 
Eine Beherrschung des Konjunktivs ist wichtig für Deutschlerner/innen. Denn der Konjunktiv stellt ein ,wichtiges Mittel der Bedeutungsschattierung“ (JUNG 1984: 233) dar. Nicht nur in der Literatur, sondern gerade auch im Alltag, etwa in Briefen, Gesprächen, Protokollen, Berichten und Erzählungen, ist eine Unterscheidung von Indikativ und Konjunktiv wichtig. Wenn diesem Unterschied nicht Rechnung getragen wird, können Missverständnisse entstehen (vgl. ebd.: 233).

Welche Funktionen und Bedeutungen kommen dem deutschen Konjunktiv als einem Mittel der Modalität im Deutschen zu? Welche Besonderheiten birgt er? Diesen Fragen geht der erste Teil dieses Artikels nach. Abschnitt 2 widmet sich den Funktionen der deutschen Verbmodi und er grenzt den Konjunktiv vom Indikativ und vom Imperativ ab. Abschnitt 3 stellt die Bedeutung des Konjunktivs als einem Mittel der Modalität heraus. Abschnitt 4 beschreibt die formalen und temporalen Besonderheiten des deutschen Konjunktivs. Die Semantik von Konjunktiv I und II wird in Abschnitt 5 und 6 beschrieben. All diese Abschnitte stützen sich auf folgende linguistische Lexika, Handbücher und Grammatiken: Althaus et al. (1980), BußMANn (2002), ConRAD (1985), Duden (2006), Duden (2009), ENGEl (1996), ENGEL (2004), GlÜCK (2000), Griesbach (1990), Heidolph et al. (1981), Helbig / Buscha (2005), HentsChel / WEYdT (2003), HoMBERGER (2000), JUNG (1984), SOMMERFELDT / STARKE (1998) und ZIFONUN et al. (1997). Bei der Auswahl war es das Ziel, aktuelle und ältere Werke zu vergleichen, um ggf. Veränderung in der Darstellung des Konjunktivs bzw. Tendenzen in der Beschreibung dessen Gebrauch zu ermitteln. Daher erklärt sich auch die Konsultation von Lexika, Handbüchern und Grammatiken aus den 1980ern und 1990ern sowie die Konsultation von mehreren Werken eines Autors.

Gerade auch aufgrund der Schwierigkeiten, die Nicht-Muttersprachler/innen mit dem deutschen Konjunktiv haben, geht dieser Artikel einer weiteren Frage nach: Wie versuchen Lern- und Übungsgrammatiken, deren Zielgruppe Deutschlerner/innen sind, den Konjunktiv darzustellen? Und welche Konsequenzen müssen aus dieser Darstellung für das Unterrichten des Deutschen als Fremdsprache gezogen werden? Diese Fragen stehen im zweiten Teil dieses Artikels im Zentrum. Abschnitt 7 untersucht, ob Lern- und Übungsgrammatiken den Konjunktiv eindeutig vom Indikativ abgrenzen, ob sie ihn als ein Mittel der Modalität beschreiben und welche Funktionen der Konjunktive im Einzelnen aufgezählt werden. Bei der Wahl der Lern- und Übungsgrammatiken wurden dabei auf Folgendes geachtet. Es wurden einerseits Grammatiken gesucht, die 
Geith, V. - Konjunktiv als Mittel der Bedeutungsschattierung

niveauspezifisch sind und daher entweder die Grund-, die Mittel- oder die Oberstufe anvisieren. Andererseits sollten Grammatiken gewählt werden, welche stufenübergreifend die deutsche Grammatik beschreiben. Folgende Grammatiken wurden gewählt:

a) für die Grundstufe: BusCHA / SZITA (2010)

b) für die Grundstufe \& Mittelstufe: DrEYER / SCHMITT (2000, 2009)

c) für die Mittelstufe: Buscha / SZITA (2011), ClAMER et al. (2006), Hering et al. (2009)

d) für die Mittel- \& Oberstufe: FAndRych (2012), Reimann (2000), Rug / TOMSASZWESKI (2009), STEIN-BASSLER (2008)

e) für die Oberstufe: BUSCHA / SzITA (2011), HALL / SCHEINER (2001)

Nach der Betrachtung dieser Lern- und Übungsgrammatiken schließt der Artikel in Abschnitt 8 mit einer Diskussion über das Unterrichten des Konjunktivs im Deutsch als Fremdsprachunterricht.

\section{Die Modi des Deutschen}

Das Deutsche besitzt drei Verbmodi: den Indikativ, den Konjunktiv und den Imperativ. Der Indikativ wird oft mit Wirklichkeitsform, der Konjunktiv mit Möglichkeitsform und der Imperativ mit Befehlsform bezeichnet. Laut Heidolph et al. beschreiben diese Begriffe die Inhalte der Modi aber nur unzureichend (vgl. HEIDOLPH et al. 1981: 521).

Der Indikativ gilt als die allgemeine Form sprachlicher Äußerungen (vgl. HeLBIG / BuSCHA 2005: 174). Er ist der unmarkierte bzw. neutrale Darstellungsmodus, der Normal- und Standardmodus (vgl. BubMANn 2000: 366, DUDEN 2009: 500). Zifonun et al. verwenden für den Indikativ sogar den Begriff default-Modus (vgl. ZIFONUN et al. 1997: 1731). Default heißt zwar Standard, kann aber auch mit Ausfall übersetzt werden. Dies ist insofern passend, als der Indikativ immer dann gebraucht wird, wenn die Verwendungsbedingungen für andere Modi nicht gegeben sind, d.h. ausfallen. Der unmarkierte Modus Indikativ wird immer dann verwendet, wenn eine Sachverhaltsbeschreibung als Tatsache präsentiert wird, oder wenn ausgedrückt werden soll, dass sie auf die bestehende Wirklichkeit zutrifft; er wird auch gebraucht, wenn Vorhersagen über eine zukünftige Wirklichkeit bzw. Versprechungen hinsichtlich ihres Eintretens gemacht 
Geith, V. - Konjunktiv als Mittel der Bedeutungsschattierung

werden (vgl. DUDEN 2009: 501). Modalausdrücke wie vielleicht, wahrscheinlich, möglicherweise können die Verbindlichkeit bzw. den Geltungsanspruch einer indikativischen Aussage allerdings relativieren (vgl. ebd.: 501). Der Duden fasst dies wie folgt zusammen:

Mit dem Indikativ und nur mit dem Indikativ kann der Sprecher sich unmittelbar auf die ,wirkliche Welt ${ }^{`}$ beziehen, Sachverhalte im Diskurs als gegeben hinstellen und die eigene Einstellung (eigenes Wissen, Glauben, Fürchten usw.) ausdrücken. Und wenn im gegebenen Zusammenhang nichts dagegen spricht, werden indikativische Verbformen auch in dem Sinn gedeutet. (ebd.: 501)

Im Gegensatz zum Indikativ ist der Konjunktiv der markierte Modus, der nur unter ganz bestimmten Voraussetzungen zu benutzen ist (vgl. ebd.: 516). Der Konjunktiv beschränkt die allgemeine Geltung einer Äußerung, denn er signalisiert, dass ihr Inhalt nicht voraussetzungslos, sondern nur unter bestimmten Bedingungen gilt. Er signalisiert ferner, dass der Sprecher sich von der beschränkt gültigen Aussage distanziert und kann deshalb als „Ausdruck der Distanzierung des Sprechers vom vollen allgemeingültigen Inhalt des Gesagten“ (HEIDOLPH et al. 1981: 521) charakterisiert werden. Der Konjunktiv kann aufgrund des Klassifikationsmerkmals > Geltungsgrad< in zwei Gruppen - in Konjunktiv I und Konjunktiv II - eingeteilt werden (vgl. Jung 1984: 226). Der Konjunktiv I umfasst die Konjunktive des Präsens, des Perfekts und des Futurs I und bringt andere Aspekte einer Äußerung zur Geltung als der Konjunktiv II, zu dem die Konjunktive des Präteritums und des Plusquamperfekts zählen. Mit dem Konjunktiv I kann u.a. eine fremde oder früher eigene Rede dargestellt sowie eine Aufforderung oder eine Bitte zum Ausdruck gebracht werden (siehe Abschnitt 5); der Konjunktiv II markiert u.a. eine Aussage als irreal, potenziell oder höflich und dient der Äußerung von Wünschen (siehe Abschnitt 6).

Der Imperativ nimmt in paradigmatischer und syntagmatischer Hinsicht eine Sonderstellung ein. Es handelt sich bei ihm um eine kategorielle Form, die das Merkmal $>$ Aufforderung < trägt (vgl. HEIDOLPH et al. 1981: 526). Mit dem Imperativ kann ein Sprecher eine direkte Aufforderung an einen (oder mehrere) Gesprächspartner richten, damit dieser (bzw. diese) den noch nicht existierenden Sachverhalt realisiert (bzw. realisieren) (vgl. HELBIG / BUSCHA 2005: 618).

\section{Modalität im Deutschen}

Der Konjunktiv ist ein Mittel der Modalität. Die Modalität gibt Aufschluss darüber, ob ein Geschehen vom Standpunkt des Sprechers aus wirklich, vorgestellt, möglich, 
Geith, V. - Konjunktiv als Mittel der Bedeutungsschattierung

vermutet, befohlen, erforderlich oder unsicher ist (vgl. JUNG 1984: 224). Sie spiegelt die Auffassung des Sprechers wider und gibt an, ob der Sprecher eine Aussage in ihrem Wirklichkeitsgehalt als eingeschränkt oder uneingeschränkt gültig verstanden wissen will. Sie informiert ferner über den Redehintergrund des Sprechers zum Sprechzeitpunkt: Sie verweist auf den Glauben, den Willen und das Wissen des Sprechers sowie auf seine Wünsche bezüglich der Wirklichkeit und seine Einstellungen hinsichtlich dessen, was gesagt wird (vgl. DUDEN 2009: 500).

Das Deutsche verfügt über eine Reihe lexikalischer, syntaktischer und morphologischer Mittel, die den Geltungsgrad einer Aussage bestimmen. Der Modus ist das morphologische Mittel der Modalität. $\mathrm{Zu}$ den Mitteln, die die Bedeutung einer Aussage bestimmen, zählen neben dem Modus auch das syntaktische Mittel der würdeForm sowie folgende lexikalische Mittel: Partikel, Modalwörter, Modalverben und die mit einem Infinitiv kombinierbaren Verben sein und haben (vgl. JUNG 1984: 224f.)

Der Modus deckt nicht das ganze Spektrum, sondern nur einen Teilbereich der Modalität ab. Seine Funktion besteht darin, den „Wirklichkeitsbezug“ bzw. den „Wahrheitsanspruch“ (DUDEN 2009: 429) einer Äußerung zu bestimmen. Ferner informiert der Sprecher durch die Wahl eines bestimmten Modus über den Geltungsanspruch einer Sachverhaltsbeschreibung, d.h. darüber, ob das Geschehen ohne Einschränkungen zu verstehen ist oder nicht (vgl. HEIDOLPH et al. 1981: 521). Der Modus ,gestattet die Färbung einer Aussage“ (HOMBERGER 2000: 342) und kann daher auch als Aussageweise des Verbes definiert werden (vgl. JuNG 1984: 225). Der Konjunktiv besitzt folglich neben der morphologischen Funktion (Bestimmung des Verbes in Person und Numerus) auch die semantische Funktion „Bedeutungsschattierung“(JUNG 1984: 233).

\section{Besonderheiten des deutschen Konjunktivs}

\section{Lückenhaftes Formenparadigma und Formensynkretismus}

Das Formenparadigma des Konjunktivs ist stark lückenhaft, denn viele Formen des Konjunktivs sind mit dem Indikativ bzw. dem Präteritum identisch (vgl. GLÜCK 2000: 365). Dies ist vor allem bei schwachen Verben der Fall. 
Geith, V. - Konjunktiv als Mittel der Bedeutungsschattierung

Wie ein Blick auf die unten stehende Tabelle zeigt, unterscheiden sich die 1. Person Singular, die 1. und 3. Person Plural schwacher Verben im Konjunktiv I nicht von denselben Personen im Indikativ Präsens. Nur bei der 2. Person Plural sowie bei der 2. und der 3. Person Singular kann man formale Differenzen feststellen. Ein Vergleich des Präteritums mit dem Konjunktiv II verdeutlicht, dass sich in keiner Person ein formaler Unterschied ergibt.

TABELLE 1 - Schwache Verben am Beispiel von lachen

\begin{tabular}{l|l|l|l}
\hline Indikativ Präsens & Konjunktiv I & Indikativ Präteritum & Konjunktiv II \\
\hline ich lache & ich lache & ich lachte & ich lachte \\
du lachst & du lachest & du lachtest & du lachtest \\
er lacht & er lache & er lachte & er lachte \\
wir lachen & wir lachen & wir lachten & wir lachten \\
ihr lacht & ihr lachet & ihr lachtet & ihr lachtet \\
sie lachen & sie lachen & sie lachten & sie lachten \\
\hline
\end{tabular}

Der Duden spricht wegen des Fehlens formaler Differenzen von „systematischen Synkretismen“ (DUDEN 2006: 442) bzw. von „morphologischer Unterbestimmtheit“ (DUDEN 2009: 432). Bei starken und gemischten Verben mit Umlaut ist dieser Synkretismus nicht so ausgeprägt, denn umlautfähige Verben erhalten einen Umlaut. Allerdings findet man auch einige übereinstimmende Formen.

TABELLE 2 - Starke Verben am Beispiel von brechen

\begin{tabular}{l|l|l|l}
\hline Indikativ Präsens & Konjunktiv I & Indikativ Präteritum & Konjunktiv II \\
\hline ich breche & ich breche & ich brach & ich bräche \\
du brichst & du brechest & du brachst & du brächest \\
er bricht & er breche & er brach & er bräche \\
wir brechen & wir brechen & wir brachen & wir brächen \\
$i$ ihr brecht & ihr brechet & ihr bracht & ihr brächet \\
sie brechen & sie brechen & sie brachen & sie brächen \\
\hline
\end{tabular}

TABELLE 3 - Gemischte Verben am Beispiel von denken

\begin{tabular}{|c|c|c|c|}
\hline Indikativ Präsens & Konjunktiv I & Indikativ Präteritum & Konjunktiv II \\
\hline ich denke & ich denke & ich dachte & ich dächte \\
\hline$d u$ denkst & du denkest & du dachtest & $d u$ dëchtest \\
\hline er denkt & er denke & er dachte & er dächte \\
\hline wir denken & wir denken & wir dachten & 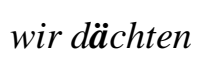 \\
\hline
\end{tabular}


Geith, V. - Konjunktiv als Mittel der Bedeutungsschattierung

\begin{tabular}{|c|c|c|c|}
\hline $\begin{array}{l}\text { ihr denkt } \\
\text { sie denken }\end{array}$ & $\begin{array}{l}\text { ihr denket } \\
\text { sie denken }\end{array}$ & $\begin{array}{l}\text { ihr dachtet } \\
\text { sie dachten }\end{array}$ & 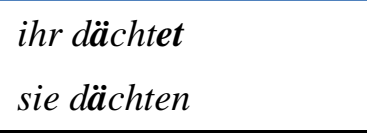 \\
\hline \multicolumn{4}{|c|}{$\begin{array}{l}\text { Auch Modalverben sind nur teilweise ,graphisch und phonisch differenziert“ } \\
\text { (ZIFONUN 1997: 1740). Im Konjunktiv II erhalten Verben mit umlautfähigen Vokal } \\
\text { einen Umlaut (mit Ausnahme von sollen und wollen) und können dadurch } \\
\text { unterschieden werden. }\end{array}$} \\
\hline \multicolumn{4}{|c|}{$\begin{array}{l}\text { Im Konjunktiv I hat nur das Verb sein eine eigene Form, die sich in allen } \\
\text { Personen vom Indikativ unterscheidet. Die Modalverben dürfen und können wechseln } \\
\text { zwar im Singular den Vokal und erhalten einen Umlaut. Allerdings gibt es in der 1. und } \\
\text { 3. Person Plural wieder Übereinstimmungen. Bei den Verben haben und werden gibt es } \\
\text { zudem Synkretismen in der 1. Person Singular. }\end{array}$} \\
\hline & TABELLE 4 - M & en am Beispiel von $k \ddot{o} n$ & \\
\hline Indikativ Präsens & Konjunktiv I & Indikativ Präteritum & Konjunktiv II \\
\hline ich kann & ich könne & ich konnte & ich könnte \\
\hline du kannst & du könnest & du konntest & du könntest \\
\hline er kann & er könne & & er könnte \\
\hline wir können & wir können & wir konnten & wir könnten \\
\hline ihr könnt & ihr könnet & ihr konntet & ihr könntet \\
\hline sie können & sie können & sie können & sie k̈̈nnten \\
\hline
\end{tabular}

\section{Die Verwendung der würde-Form}

Treten Fälle von Synkretismus bei Konjunktiv-I-Formen auf, so werden sie durch den Konjunktiv II ersetzt. Uneindeutige Konjunktiv-II-Formen werden durch die würdeForm ausgetauscht (vgl. GRIESBACH 1990: 96). So lautet zumindest eine „sprachpflegerische Empfehlung“ (ZIFONUN et al. 1997: 1784). Allerdings spiegelt diese Empfehlung nicht die Sprachwirklichkeit wieder (vgl. ebd. 1784). Denn die analytisch gebildete würde-Form ersetzt häufig eigentlich eindeutige synthetische Konjunktivformen. Dies gilt in erster Linie für Formen des Konjunktivs II von vielen starken Verben, da diese gehoben, altertümlich oder gespreizt wirken und ihr Gebrauch normalerweise umgangen wird. Einige Beispiele hierfür sind: bärge, drösche, flöchte, flöhe, genösse etc. (vgl. ALtHAus et al. 1980: 625). 
Aber auch ein Ersatz des Konjunktivs I ist üblich, selbst wenn die Formen eindeutig unterscheidbar wären (vgl. ebd.: 625). Denkt man etwa an die Formen du denkest, ihr denket, $d u$ brechest, ihr brechet aus den oben stehenden Tabellen, kann man nachvollziehen, warum diese im heutigen Deutsch tendenziell entweder durch die entsprechende Konjunktiv-II-Form oder die würde-Form ersetzt werden. Denn auch diese Konjunktiv-I-Formen wirken eher veraltet.

Im Schriftsprachlichen kann man eine Tendenz zum Ersatz der synthetischen Konjunktivformen feststellen. Diese Tendenz verstärkt sich in der gesprochenen Sprache. Denn hier werden häufig nur die Konjunktivformen von sehr gebräuchlichen Verben verwendet. $\mathrm{Zu}$ diesen Verben gehören: haben, sein, werden, kommen, gehen, wissen, geben, tun, gebrauchen, finden, bringen, können, sollen, wollen, dürfen, mögen und müssen (vgl. ZIFONUN et al. 1997: 1784). Laut Duden ist die Entwicklung in der gesprochenen Sprache so weit vorangeschritten, dass man mit Ausnahme dieser sehr geläufigen Verben die würde-Form als die Normalform bei Vollverben ansehen kann (vgl. DudEN 2009: 540): „Der Konjunktiv (II) kommt als Konjugationskategorie im Normalfall nicht am einfachen Vollverb, sondern erst im mehrgliedrigen Verbalkomplex morphologisch zum Ausdruck.“" (ebd. 2009: 540)

Allerdings gilt es anzumerken, dass ein Ersatz von synthetischen Konjunktivformen nicht immer automatisch geschieht. Dies gilt bei zusammengesetzten Konjunktivformen. Der Satz An deiner Stelle hätte ich ihn gefragt. wird eher selten durch eine würde-Form zu An deiner Stelle würde ich ihn gefragt haben. Vollkommen ungebräuchlich ist ein solcher Ersatz in der indirekten Rede (vgl. HELBIG / BUSCHA 2005:172).

Ferner sei darauf verwiesen, dass der Ersatz von Konjunktiv II durch die würdeForm nur bei perfektiven, nicht jedoch bei durativen Verben üblich ist (vgl. ENGEL 1996: 424).

(1) Sie schrieb, sie würde mit dem Rauchen aufhören. (perfektives Verb: würde-Ersatz üblich) (vgl. ebd.: 424)

(2) Sie schrieb, sie würde im Bett liegen. (duratives Verb: würde-Ersatz unüblich) (vgl. ebd.: 424)

Auf die würde-Form wird ferner verzichtet, wenn der Kontext unzweideutig ist, oder wenn Temporalangaben vorliegen (vgl. GRIESBACH 1990: 96) 
Geith, V. - Konjunktiv als Mittel der Bedeutungsschattierung

(3) Wenn ich so wenig Geld hätte wie Karl, kaufte ich mir kein neues Auto. (ebd.: 96)

(4) Wir gingen morgen ins Kino, wenn uns nicht gerade unsere Eltern besuchen wollten. (ebd.: 96)

\section{Fehlende temporale Differenzierung und temporale Synonymie}

Der Indikativ verfügt über ein sechsgliedriges Tempussystem. Innerhalb dieses Systems gibt es drei Zeiten, die einen Vergangenheitsbezug ausdrücken können: Präteritum, Perfekt und Plusquamperfekt. Sie stehen dem Präsens gegenüber, das auf NichtVergangenes hinweist. Ein Geschehen mit Zukunftsbezug kann durch zwei Zeiten (Futur I und II) ausgedrückt werden.

Im Konjunktiv dagegen existiert nur ein zweigliedriges Tempussystem. Ein Vergangenheitsbezug wird durch die zusammengesetzten Zeiten der Konjunktive (sei gelaufen, wäre gelaufen) dargestellt. Ein Bezug zur Gegenwart oder Zukunft wird mit den einfachen Zeiten der Konjunktive (laufe, liefe) oder mit der würde-Form (würde laufen) ausgedrückt. In der Folge kommt es zu temporalen Synonymien. Laufe, liefe und würde laufen, sofern die würde-Form als Ersatzform gebraucht ist, können nicht temporal unterschieden werden.

Der Unterschied zwischen Gegenwärtigem und Vergangenem bzw. zwischen Gegenwärtigem und Zukünftigem kann zwar auch im Konjunktiv mithilfe der zusammengesetzten Zeiten ausgedrückt werden. Aber da diese zusammengesetzten Konjunktive jene Funktionsbereiche einschließen, die im Indikativ auf drei Vergangenheitsstufen verteilt sind, fehlen hier die Möglichkeiten zur temporalen Differenzierung (vgl. DUdEN 2009: 500, 517; HeIDOLPH et al. 1981: 522f.). Aufgrund des Fehlens dieses formalen Kennzeichens für die Vorzeitigkeit muss diese aus dem Inhalt der Aussage selbst erschlossen werden (vgl. HeLBIG / BusCHA 2005: 171).

(5) Wenn es in der Nacht geregnet hatte, goss sie am Morgen die Blumen nicht. (verschiedene Tempusformen stehen für verschiedene Zeiten) (ebd.: 171)

(6) Wenn es in der Nacht geregnet hätte, hätte sie am Morgen die Blumen nicht gegossen. (gleiche Tempusformen stehen für verschiedene Zeiten) (ebd.: 171)

Darüber hinaus gilt es anzumerken, dass es im schriftsprachlichen Konjunktiv kein Äquivalent zum indikativischen Plusquamperfekt (Ich war geschwommen) gibt. Zwar kann 
Geith, V. - Konjunktiv als Mittel der Bedeutungsschattierung

man im Mündlichen das doppelte Perfekt (Ich wäre geschwommen gewesen) verwenden, es gehört aber nicht zum Standard der Schriftsprache (vgl. DUDEN 2009: 517).

Erschwerend kommt hinzu, dass zusammengesetzte Zeiten nicht immer auf ein vergangenes Geschehen hinweisen, etwa bei einem Satz dieser Art:

Im kommenden Jahr wäre Goethe 268 Jahre alt geworden.

In diesem Fall bezieht sich der Konjunktiv des Plusquamperfekts auf ein zukünftiges Geschehen (Geburtstag von Goethe), dessen Realisierung aber gleichzeitig von einem Ereignis in der Vergangenheit verhindert wird (Tod Goethes) (vgl. ebd.: 518).

\section{Semantik des Konjunktivs I}

„In der Schriftsprache ist das Referat bzw. die indirekte Rede (indirekte Redewiedergabe der wichtigste Funktionsbereich des Konjunktivs. Zählungen bestätigen, dass er hier am häufigsten auftritt.“ (DUDEN 2009: 523). Die linguistischen Lexika, Grammatiken und Handbüchern teilen diesen Blick des Dudens auf die Hauptfunktion des Konjunktivs I. Zwar variieren die Bezeichnungen. Der DuDEN (2009: 523) beispielsweise bezeichnet den Konjunktiv in der indirekten Rede als „Indirektheitskonjunktiv“, bei EISENBERG (2006: 120) heißt er „Referatskonjunktiv“ und bei CONRAD (1985: 126) „obliquer Konjunktiv.“ Aber trotz der unterschiedlichen Bezeichnungen wird er durchgängig von allen untersuchten Autoren erwähnt und ähnlich beschrieben.

Große Uneinigkeit dagegen besteht, was die Bezeichnung, Kategorisierung und Definition der anderen Funktionen des Konjunktivs I angeht. Der DUDEN (2009: 536) fasst alle diese anderen Funktionen unter einem Begriff zusammen und bezeichnet den Funktionsbereich als volitiven Konjunktiv. Diese Bezeichnung ist ein Sammelbegriff und vereint sehr unterschiedliche Sprechhandlungen wie Wünsche, Bitten und Aufforderungen. SOMMERFELDT und STARKE verwenden einen ähnlichen Begriff, den voluntativen Konjunktiv. Auch bei ihnen dient er als Sammelbecken für sehr unterschiedliche sprachliche Handlungen wie Wünsche, Absichten, Ratschläge und Empfehlungen (vgl. 1998: 77). Auch EISENBERG verwendet diese Bezeichnung des volitiven Konjunktivs. Er spricht in diesem Zusammenhang allerdings nur von 
Setzungen eines Sachverhaltes und Aufforderung zu dessen Realisierung (vgl. 2006: 120). ConRAD verwendet die beiden Begriffe Volitiv und Voluntativ synonym. Bei ihm sind sie jedoch nicht Überbegriffe, sondern drücken nur den Willen bzw. die Absicht des Sprechers aus (vgl. 1985: 270). Laut HEIDOLPH et al. schließlich tritt der voluntative Konjunktiv in Losungen und feststehenden Wendungen auf (vgl. 1981: 533).

Neben dem Sammelbegriff Volitiv / Voluntativ findet man noch eine Reihe weiterer, enger gefasster Begriffe. CONRAD beispielsweise führt in Hinblick auf den Konjunktiv I noch die Bezeichnungen heischender Konjunktiv, konzessiver Konjunktiv und potenzieller Konjunktiv auf (vgl. 1985: 126). Ein anderes Beispiel sind HENTSCHEL und WEYDT, welche die Bezeichnungen Optativ und Adhortativ verwenden (vgl. 2003: 116).

Problematisch an dieser Begriffsvielfalt ist, dass oft gleiche Begrifflichkeiten auf verschiedene Arten definiert werden. Als Beispiel hierfür dient nicht nur der oben bereits erwähnte voluntative Konjunktiv. Ähnliches gilt für den adhortativen Konjunktiv. Während er bei HENTSCHEL und WEYDT (vgl. 2003: 116) als Ersatzform für den Imperativ dient, wird er von ENGEL (vgl. 1996: 419, 2004: 218) als Sammelbegriff verwendet und erstreckt sich in der Konsequenz auf vielfältige Kontexte.

Diese Begriffs- und Definitionsvielfalt verdeutlicht, dass der Funktionsbereich des Konjunktivs I nicht übereinstimmend und noch nicht abschließend definiert wurde. Dies mag damit zusammenhängen, dass die Funktionen des Konjunktivs I oft nicht eindeutig zu trennen sind (vgl. DUDEN 2009: 536).

Daher wird auch im Folgenden der Konjunktiv I wie folgt beschrieben. Er wird in drei Funktionsbereiche aufgeteilt. Der Funktionsbereich 1 wird in Anlehnung an den DUDEN (2009) als Indirektheitskonjunktiv bezeichnet. Der Funktionsbereich 2 umfasst Wünsche, Bitten, Aufforderungen, Ratschläge, Empfehlungen sowie Zugeständnisse und Einräumungen und er wird ebenfalls in Analogie an den Duden mit Volitiv betitelt. Auf eine weitere terminologische Unterteilung dieses Funktionsbereichs wird verzichtet. Aber auch wenn der Konjunktiv I begrifflich nicht weiter unterteilt wird, so werden doch innerhalb dieses Funktionsbereiches die einzelnen Verwendungsweisen expliziert. Neben diesen ersten beiden Funktionsbereichen, in deren Mittelpunkt Sprachhandlungen stehen, soll noch ein dritter Funktionsbereich aufgemacht werden. In 
ihm wird eine Verwendungsweise des Konjunktivs I gesammelt, die keiner reinen Sprachhandlung zugeordnet werden konnte.

\section{Funktionsbereich 1: Indirektheitskonjunktiv}

Will man etwas wiedergeben, das $\mathrm{zu}$ einem früheren Zeitpunkt von einem anderen Sprecher oder der eigenen Person gesagt wurde, so hat man zwei Möglichkeiten: die direkte oder die indirekte Rede. Bei Verwendung direkter Rede kann der Sprecher das Gesagte in genau der Formulierung reproduzieren, in der es ursprünglich war.

Entscheidet sich der Sprecher für die indirekte Rede, kann er entweder den Indikativ oder den Konjunktiv als Modus wählen. Helbig und Buscha sind der Meinung, dass der Konjunktiv bei der Wiedergabe fremder Rede ein fakultatives Mittel ist. Es reiche, dass mindestens eines der formalen Mittel (redeeinleitendes Verb, Nebensatzform, Konjunktiv) vorhanden ist (vgl. HeLBIG/BUSCHA 2005: 174). Demgegenüber ist für Engel nur ein im Konjunktiv stehender Satz ein formal eindeutig markierter Satz der indirekten Rede, denn er zeigt eindeutig an, dass eine Rede wiedergeben wird (unter der Annahme, dass der Ausgangssatz keine hypothetische Äußerung enthielt). Die kann man an folgenden Beispielen ablesen.

(8) Maria sagte, dass sie heute um 18 Uhr zu uns kommt.

(9) Maria sagte, dass sie heute um 18 Uhr zu uns komme.

Der erste Satz drückt die Meinung des Sprechers aus. Diese Meinung stimmt zwar in diesem Fall mit der von Maria überein, aber möglicherweise kann man sie nicht unmittelbar auf eine Äußerung Marias zurückführen (vgl. ENGEL 1996: 423). Der Konjunktiv einer indirekten Rede signalisiert Distanz in zweifacher Weise. Mit der formalen Kennzeichnung einer Rede als fremder oder früher eigener Rede distanziert sich der Sprecher gegenüber dem beschriebenen Sachverhalt. Er zeigt auf, dass der Sachverhalt nicht als ursprünglich oder unmittelbar gesetzt, sondern nur als vermittelt verstanden werden darf. Zum anderen wird verdeutlicht, dass zwischen dem Urheber des Redeakts und dem Hörer eine kommunikative Distanz besteht, denn schließlich ist der Sprecher als eine weitere Instanz bei der Vermittlung der Rede zwischen sie getreten (vgl. HEIDOLPH et al. 1981: 524). 


\title{
Funktionsbereich 2: Volitiv
}

\author{
Volitiv zur Äußerung von Wünschen und Bitten
}

Direkte Wünsche und Bitten in Verbindung mit Konjunktiv I werden häufig in Verbindung mit den Verben sein, mögen und wollen geäußert. Gelegentlich kommen auch Vollverben in solchen Sätzen vor (vgl. DUDEN 2009: 537).

(10) Dem Autor sei Dank! Man möge verstehen oder verurteilen! (DUDEN 2009: 537)

(11) Es sage uns niemand, heute gebe es keine sachliche Alternative mehr. [AUGSTEIN o.A. zitiert in DUDEN 2009: 537)

(12) Es lebe das Geburtstagskind! (HENTSCHEL / WEYDT 2003: 116)

(13) Möge jeder sein Bestes tun! (JUNG 1984: 228)

(14) Es lebe der erste Mai! (HEIDOLPH et al. 1981: 533)

(15) Gott segne dich! Gott sei Dank! (GRIESBACH 1990: 97)

Auch indirekt geäußerte Wünsche und Bitten können mit dem Konjunktiv I stehen. Der Duden nennt hier folgende Beispiele:

(16) Seine Aufforderung, sie möge ihm zuhören, stieß auf taube Ohren. Der Onkel verlangte nun, dass Jonathan sich unverzüglich vereheliche (...) [SÜßKIND o.A. zitiert in DUDEN 2009: 537]

In diese Kategorie der indirekt geäußerten Wünsche und Bitten können auch finale Nebensätze mit Konjunktiv I eingeordnet werden, denn im Nebensatz wird dargestellt, welche Absichten verfolgt bzw. welche Wünsche realisiert werden sollen.

(17) [...] du wirst [...] um Hilfe schreien, damit man mit Leitern komme und dich vor einer Taube rette, vor einer Taube! [SÜßKIND o.A. zitiert in DUDEN 2009: 537]

(18) Sie beten, dass das Dorf von der Besetzung verschont bleibe. (ENGEL 1996: 420)

(19) Er schickte den Kindern zwei Leute nach, damit ihnen auf dem weiten Weg nichts geschehe. (ebd. 420)

In Finalsätzen können sowohl Konjunktiv I als auch Konjunktiv II vorkommen (vgl. GRIESBACH 1990: 99). Der Gebrauch des Konjunktivs in solchen Sätzen ist nur fakultativ (vgl. HEIDOLPH et al. 1981: 535). HEIDOLPH et al. stellten schon im Jahr 1981 eine Tendenz zum Gebrauch des Indikativs in Finalsätzen fest (vgl. ebd.: 535). Der Konjunktiv in finalen Sätzen wird bei ENGEL (1996) und GRIESBACH (1990) erwähnt. Die anderen untersuchten jüngeren Lexika, Handbücher und Grammatiken gehen auf 
Geith, V. - Konjunktiv als Mittel der Bedeutungsschattierung

den Konjunktiv I in Finalsätzen nicht ein. Es scheint, dass sich die von HEIDOLPH et al. beobachtete Tendenz verstetigt hat und dass heutzutage der Indikativ dem Konjunktiv in Finalsätzen vorgezogen wird. Auch der Duden schreibt bezüglich dieser Sätze: „Der Normalmodus ist jedoch heute der Indikativ, vor allem in präsentischen Kontexten.“ (DUDEN 2009: 538).

Volitiv zur Äußerung von Aufforderungen, Ratschlägen und Empfehlungen

Der Konjunktiv tritt auch bei Aufforderungen, Ratschlägen und Empfehlungen auf. Das Subjekt solcher Aufforderungen kann die 3. Person Singular sein. Der in solchen Sätzen geäußerte Appell richtet sich nicht direkt an einen Gesprächspartner. Er ist daher nur mittelbar, im Gegensatz zu einer Aufforderung im Imperativ, mit der immer unmittelbar an Gesprächspartner appelliert wird (vgl. HEIDOLPH et al. 1981: 533).

(20) Er komme herein! (HEIDOLPH et al. 1981: 533)

(21) Aber da bringe ihn mal einer von ab! (ebd.: 533)

Aufforderungen im Konjunktiv können darüber hinaus auch in der 1. oder der 3. Person Plural geschehen. Sie wirken zumeist höflicher.

(22) Seien wir doch vernünftig! Seien Sie mir bitte nicht böse! (DUDEN 2009: 537)

Aufforderungen mit Konjunktiv treten ferner mit dem Pronomen man auf, d.h. sie sind an keine bestimmte Person gerichtet. Diese Verbindung aus Konjunktiv I und unpersönlicher Aufforderung ist typisch für bestimmte Textsorten wie Anleitungen, Gebrauchsanweisungen und Rezepte. Allerdings muss man hier anmerken, dass der Konjunktiv in diesem Zusammenhang immer häufiger durch den Infinitiv ersetzt wird (vgl. Duden 2009: 537, ENGEL 2004: 218).

(23) Man nehme fünf Eier Mehl, dann rühre man das Ganze gut durch. (DUDEN 2009: 537)

(24) Man behandle die Bienen nur mit sauberen Händen und sauberen Gerätschaften, benutze möglichst keine Abkehrbesen ... (HEIDOLPH et al. 533)

(25) Die Bäume schneide man, bevor man sie ausschlage. (ENGEL 2004: 218)

Eine andere Art der Aufforderung, die ebenfalls textsortenspezifisch ist, findet man bei (mathematischen, physikalischen) Fachtexten. 
(23) In der Zeichnung sei die Ellipse eine Planetenbahn. (DUDEN 2009: 537)

(24) Gegeben sei ein gleichschenkliges Dreieck mit der Seite a $=12 \mathrm{~cm}$. (ENGEL 2004: 218)

Volitiv zum Ausdruck eines Zugeständnisses oder einer Einräumung

Ferner kann der Konjunktiv in konzessiven Nebensätzen erscheinen. Konzessivsätze implizieren immer eine Einräumung, d.h. sie drücken immer eine nicht-entscheidende Bedingung oder einen unzureichenden Gegengrund aus (vgl. ENGEL 1996: 420). Wie die folgenden Beispiele zeigen tritt der Konjunktiv I bei Zugeständnissen und Einräumungen in Nebensätzen auf, die zu formelhaften Wendungen geworden sind:

(25) Sei es in diesem Jahr oder im nächsten, einmal werden wir euch besuchen kommen. (GRIESBACH 1990: 98)

(26) Wie dem auch sei, wir werden uns von unserem Vorhaben nicht abbringen lassen. (ebd. 1990: 98)

In solchen Konzessivsätzen kann auch der Indikativ stehen. Allerdings wirkt er sich auf die Realität der Aussage aus. Der indikativische Satz beschreibt eine „einzuräumende Tatsache“ (CONRAD 1985: 126). Der Indikativ hebt die Bedeutung des Satzes sozusagen auf die Ebene der Wirklichkeit zurück. Dies lässt sich an folgenden Beispielen ablesen:

(27) Ich bin glücklich, was da auch kommen möge. (ebd.: 126)

(28) Ich bin glücklich, was da auch kommen mag. (ebd.: 126)

Funktionsbereich 3: Vorsichtige Umschreibung des Autorenplurals

Eine letzte Verwendungsweise des Konjunktivs I konnte keiner der beiden vorausgegangenen Funktionsbereiche zugeordnet werden. In wissenschaftlichen Texten treten häufig Formulierungen wie in folgenden Sätzen auf:

(29) Es sei hier nur die Vieldeutigkeit des Präfixes ver- erwähnt... (HELBIG / BUSCHA 2005: 184)

(30) In diesem Zusammenhang sei betont, dass ... (ENGEL 1996: 420)

Solche Konstruktionen erfüllen zwei Funktionen. Sie ersetzen erstens den Autorenplural. Denn wie man an dem ersten Beispielsatz sehen kann, ist eine passivische Konstruktion in Verbindung mit dem Konjunktiv an die Stelle eines Subjekts und eines Modalverbs getreten, das einen Willen ausdrückt: „Wir möchten hier nur die Vieldeutigkeit des Präfixes ver- erwähnen...“ (HELBIG / Buscha 2005: 184) 
Darüber hinaus sind solche Konstruktionen vorsichtiger. Vorstellbar wäre nämlich auch ein Satz im Indikativ. Dieser hieße Analogie zu dem zweiten Beispielsatz: „In diesem Zusammenhang ist zu betonen, dass ..." (ENGEL 1996: 420). Im Gegensatz zum Konjunktiv signalisiert der Indikativ jedoch „stärkere Notwendigkeit“ (ebd.: 420).

\section{Semantik des Konjunktivs II}

Verwendet ein Sprecher den Konjunktiv II, so verweist er darauf, dass die von ihm geäußerte Sachverhaltsbeschreibung nur eingeschränkte Gültigkeit besitzt und dass sie nicht als wirklich verstanden werden soll, weil sie sich nicht auf eine objektive Realität, sondern nur auf eine fiktive, in der Vorstellung des Sprechers existierende Ebene bezieht (vgl. HEIDOLPH et al. 1981: 525f.). Durch den Gebrauch des Konjunktivs II distanziert sich der Sprecher ,ausdrücklich von einem normalerweise zu erwartenden allgemeinen Wirklichkeitsbezug“ (ebd.: 525). Dass ein Satz im Konjunktiv einem normalerweise zu erwartenden Wirklichkeitsbezug entgegenstehen kann, sieht man schön an folgendem Beispiel:

[...] jedenfalls flog ich beinahe, und hätte ich nur meinen Mantel aufgeknöpft und beide Hälften in die Hände genommen und wie Flügel ausgebreitet, dann hätte mich der Wind vollends emporgehoben [...]. Aber ich habe den Mantel nicht aufgeknöpft und bin nicht wirklich hoch hinaufgeflogen. (SÜSKIND o.A. zitiert in DUDEN 2009: 517).

Das, was man normalerweise erwarten würde (Fliegen ist nicht möglich), steht im Indikativ. Im durch den Konjunktiv begründeten gedanklichen Konstrukt erscheint dies jedoch realisierbar. Inhaltlich drückt eine durch den Konjunktiv II in ihrer Gültigkeit beschränkte Aussage Irrealität (Kontrafaktizität, Nichtwirklichkeit) oder Potenzialität (Eventualität, Möglichkeit) aus. Diese Dimensionen treten häufig in Zusammenhang mit verschiedenen Nebensatzgefügen (Komparativ-, Konzessiv-, Konsekutiv- und Konditionalsätzen) auf. Daneben erscheinen Irrealität und Potentialität in verkappten oder selbstständigen Nebensätzen sowie in Nebensätzen, die auf negierte Hauptsätze folgen. Und schließlich verwendet man Konjunktiv II auch, um höflich $\mathrm{zu}$ sein, und in Verbindung mit Modalverben. 


\section{Irrealer Vergleich: Konjunktiv II in Komparativsätzen}

Irreale Komparativsätze werden durch die Subjunktoren als wenn, wie wenn und als ob hergestellt. Sie drücken eine hypothetische, nicht reale, sondern gedanklich vorgestellte Gleichheit aus. In diesen Komparativsätzen ist der Konjunktivgebrauch fakultativ. Er kann durch den Indikativ ersetzt werden, ohne dass sich die Bedeutung wesentlich verändert (vgl. HELBIG / BUSCHA 2005: 180).

(31) Irrealer Komparativsatz mit Konjunktiv:

Tom sah aus, als ob er die ganze Nacht nicht geschlafen hätte.

(32) Ersatz des Konjunktivs durch den Indikativ:

Tom sah aus, als ob er die ganze Nacht nicht geschlafen hat.

Irreale Vergleichsätze stehen hauptsächlich mit dem Konjunktiv II, sind aber auch mit dem Konjunktiv I denkbar. Der Satz aus dem Beispiel hieße dann:

(33) Tom sah aus, als ob er die ganze Nacht nicht geschlafen habe.

\section{Hypothetische oder irreale Einräumung: Konjunktiv II in Konzessivsätzen}

Konzessivsätze drücken eine Einräumung, ein Zugeständnis, eine unzureichende Bedingung oder einen nicht wirksamen Sachverhalt aus. Sie können real, hypothetisch oder irreal sein. Reale Konzessivsätze stehen im Indikativ und werden durch Subjunktoren (z.B. obwohl) oder Konjunktionaladverbien (z.B. trotzdem) eingeleitet (vgl. HeLBIG / BuSCHA 2005: 183). Irreale und hypothetische Konzessivsätze gehen mit den Subjunktoren wenn auch und auch wenn einher. Zusätzlich kann in den Hauptsätzen ein so ... doch erscheinen, sofern der Neben- dem Hauptsatz vorangeht.

(34) Realer Konzessivsatz:

Das Wetter ist schön. Trotzdem kann ich nicht schwimmen gehen.

(35) Hypothetischer Konzessivsatz:

Ich könnte nicht schwimmen gehen, auch wenn das Wetter schön wäre.

Auch wenn das Wetter schön wäre, (so) könnte ich (doch) nicht schwimmen gehen.

(36) Irrealer Konzessivsatz:

Auch wenn das Wetter schön gewesen wäre, (so) hätte ich (doch) nicht schwimmen gehen können.

Hypothetische und irreale Konzessivsätze unterscheiden sich hinsichtlich der Realisierbarkeit des geschilderten Sachverhalts. Während der Sachverhalt 
Geith, V. - Konjunktiv als Mittel der Bedeutungsschattierung

hypothetischer Konzessivsätze in der Vorstellung des Sprechers verwirklicht werden kann, ist dies bei irrealen Konzessivsätzen nicht mehr der Fall. Der Duden merkt in Hinblick auf irreale Konzessivgefüge an, dass die Irrealität des Nebensatzes sich nicht immer auf den Hauptsatz übertragen muss und begründet dies an folgendem Beispiel:

Auch wenn Goethe das Jahr 1832 überlebt hätte, wäre er heute tot. “ (DUDEN 2009: 519)

Der Sachverhalt des Nebensatzes wird durch den Konjunktiv und den Subjunktor als unzutreffend charakterisiert (Goethe hat das Jahr 1832 nicht überlebt). Diese Irrealität bleibt aber ohne Folgen für den Hauptsatz, denn der Sprecher behauptet nicht, dass dieser Hauptsatz unzutreffend ist (Goethe lebt.), sondern dass er zutrifft (Goethe ist heute tot.) (vgl. ebd.: 519).

\section{Irreale Folge: Konjunktiv II in Konsekutivsätzen}

Ein irrealer Konsekutivsatz wird mit dem Subjunktor als dass gebildet und unterscheidet sich dadurch von einem realen Konsekutivsatz, der mit dem Subjunktor so dass einhergeht. Irreale Konsekutivsätze können deswegen auch mit dem Indikativ stehen, weil die Irrealität hinreichend durch den Subjunktor als dass gekennzeichnet ist. Es gibt ferner negative irreale Konsekutivsätze, die mit dem Subjunktor ohne dass gebildet werden (vgl. HELBIG / BusCHA 2005: 183).

(38) Realer Konsekutivsatz:

Das Wetter ist schlecht, so dass ich nicht schwimmen gehen kann.

(39) Irrealer Konsekutivsatz:

Das Wetter ist zu schlecht, als dass ich schwimmen gehen könnte.

(40) Negativer irrealer Konsekutivsatz:

Er ging schwimmen, ohne dass er mir Bescheid gegeben hätte.

Auch Sätze mit dass nach zu oder nach nicht so können unter irrealen Konsekutivsätzen aufgelistet werden (vgl. DUDEN 2009: 522).

(41) Ich bin nicht so betrunken, dass ich mich von Ihnen ausfragen ließe. (ebd.: 522) 


\section{Hypothetische oder irreale Bedingung: Konjunktiv II in Konditionalsätzen}

Konditionalsätze werden mit dem Subjunktor wenn eingeleitet. Im Gegensatz zu Komparativ- und Konsekutivsätzen, in denen der Konjunktiv ohne wesentliche Bedeutungsveränderung durch den Indikativ ausgetauscht werden kann, ist dies bei Konditionalgefügen nicht möglich. Denn der Konjunktiv geht mit einer hypothetischen oder irrealen Bedingung einher, während der Indikativ eine potenzielle Bedingung darstellt. Ein Ersatz des Konjunktivs durch den Indikativ würde die Geltung der Aussage wesentlich verändern.

(42) Potenzieller Konditionalsatz: Wenn die Sonne scheint, gehen wir schwimmen.

(43) Hypothetischer Konditionalsatz: Wenn die Sonne schiene, gingen wir schwimmen.

(44) Irrealer Konditionalsatz: Wenn die Sonne geschienen hätte, wären wir schwimmen gegangen.

Der potenzielle Konditionalsatz drückt aus, dass das Subjekt des Satzes das beschriebene Geschehen in der Gegenwart oder in der Zukunft verwirklicht. Der hypothetische Bedingungssatz dagegen impliziert, dass das bedingende Geschehen (Sonnenschein) und das bedingte Geschehen (schwimmen) zumindest in der gedanklichen Vorstellungswelt des Sprechers möglich oder wahrscheinlich ist. Im irrealen Konditionalsatz schließlich wird verdeutlicht, dass sowohl bedingendes als auch bedingtes Geschehen nicht verwirklicht wurden und dass dies auch nicht mehr eintreten wird (vgl. HELBIG / BUSCHA 2005: 180f.). Streng genommen ist in irrealen Konditionalsätzen nur das bedingende Geschehen kontrafaktisch. Da aber die Bedingung nicht erfüllt wird, bleibt auch das bedingte Geschehen, unverwirklicht und ist irreal (vgl. DUDEN 2009: 518).

\section{Verkappte Konditionalsätze und irreale Aussagesätze}

Hypothetische oder irreale Bedingungen werden oft nicht explizit in Form von Konditionalsätzen dargestellt. Sie treten häufig in Form von verkappten Konditionalgefügen auf, die aus dem Kontext erschlossen werden müssen. Denn sie verstecken sich hinter Infinitiv- und Partizipialkonstruktionen, Satzverbindungen mit 
Geith, V. - Konjunktiv als Mittel der Bedeutungsschattierung

aber (bzw. vergleichbaren antonymischen Sätzen im Indikativ) oder präpositionalen Gruppen.

(45) Verkappter Nebensatz mit Infinitivkonstruktion: Es wäre gut ihn zu fragen. (Es wäre gut, wenn Sie ihn selbst fragen würden.) (HELBIG / BUSCHA 2005: 182)

(46) Verkappter Nebensatz mit Partizipialkonstruktion: Über die Zeit befragt(,) wüsste ich keine Antwort. (Wenn ich über die Zeit befragt würde, wüsste ich keine Antwort.) (ebd.: 182)

(47) Verkappter Nebensatz mit aber: Ich hätte ihm geschrieben, aber ich wusste seine Adresse nicht. (Ich hätte ihm geschrieben, wenn ich seine Adresse gewusst hätte.) (ebd.: 182)

(48) Verkappter Nebensatz mit präpositionaler Gruppe: Bei intensiver Bodenbearbeitung (Wenn der Boden intensiv bearbeitet würde,) lägen die Erträge höher. (ebd.: 182)

Anzumerken gilt hier, dass in diesen Fällen auch der Indikativ stehen kann, allerdings mit einem feinen Bedeutungsunterschied. Betrachtet man etwa das letzte Beispiel der Präpositionalphrase, wäre auch folgender Satz denkbar: Bei intensiver Bodenbearbeitung liegen die Erträge höher. Dieser indikativische Satz impliziert weniger Zweifel an der Erfüllung der Bedingung als der Satz im Konjunktiv.

Helbig und Buscha verweisen ferner darauf, dass es Sätze gibt, die nur noch bedingt auf Konditionalsätze zurückgeführt werden können und dass in diesen Sätzen der Konjunktiv durch den Indikativ austauschbar ist, ohne dass sich dadurch ein erkennbarer Bedeutungsunterschied ergeben würde (vgl. HELBIG / BusCHA 2005: 182). Ein Beispiel hierfür ist etwa der folgende Satz:

(49) Es wäre nur noch das Make-up ein wenig aufzufrischen(, wenn Sie damit einverstanden wären.) (ebd.: 182)

Der Konjunktiv kann darüber hinaus auch auftreten, ohne dass er auf implizit zugrunde liegende Konditionalsätze zurückführbar wäre. Er kann bedingt sein durch satzeinleitende Wendungen (Angenommen..., Stellen Sie sich vor...) oder adverbiale Ausdrücke, wie in folgendem Satz: ,[...] ich erinnere mich genau, dass ich um ein Haar geflogen wäre“ (SÜSKIND o.A. zitiert in DUDEN 2009: 520). Solche Sätze schaffen einen nur gedachten, vorgestellten, nicht faktischen Hintergrund (vgl. ebd.: 520). 
Geith, V. - Konjunktiv als Mittel der Bedeutungsschattierung

Erfüllbarer und unerfüllbarer Wunsch: Heischender Konjunktiv in selbständigen Nebensätzen

Die Heischeform mit Konjunktiv II tritt in sogenannten selbstständigen Nebensätzen auf. Diese entstehen, wenn ein konditionales Bedingungsgefüge aufgelöst wird. Übrig bleibt dann nur der durch wenn eingeleitete Nebensatz, oder ein Satz mit Spitzenstellung des finiten Verbes. Fakultativ können die Partikeln doch, bloß und nur hinzutreten.

(50) Wenn Tom (doch / bloß / nur) schon hier wäre!

(51) Wäre Tom (doch / bloß/ nur) schon hier!

Die so entstandenen Wunschsätze können noch realisierbar sein. Ob dies der Fall ist, ist aus dem jeweiligen Kontext ersichtlich. Wenn etwa Maria auf Tom wartet und ausruft Wäre Tom doch schon hier!, dann handelt es sich hierbei um einen erfüllbaren Wunsch mit Gegenwartsbezug bzw. mit Bezug auf eine nahe Zukunft, da die Ankunft von Tom bevorsteht. Wenn Tom sich aber verspätet und Maria dann sagt Wäre Tom doch schon vor einer Stunde gekommen! Wir hätten schwimmen gehen können, so ist ersichtlich, dass der Wunsch irreal ist, weil er sich auf die Vergangenheit bezieht und nicht mehr erfüllbar ist (vgl. HEIDOLPH et al. 1981: 533).

In selbstständigen Wunschsätzen kann der Indikativ mit dem Konjunktiv konkurrieren. Vorstellbar wäre etwa folgender Satz: „Wenn sie nur zurückkommt!“ (ENGEL 1996: 424) Ein solcher Wunschsatz ist immer gekoppelt an den Subjunktor wenn sowie an die Partikel nur und er hat im Vergleich zu einem Satz im Konjunktiv stärkeren Wirklichkeitscharakter. Der Sprecher ,,ist zuversichtlicher, er glaubt mehr an die Realisierung des Sachverhalts.“ (ebd.: 424).

\section{Nebensätze nach negierten Hauptsätzen}

Der Duden erwähnt eine weitere Verwendung des Konjunktivs II. Er kann in Relativsätzen oder anderen Nebensätzen erscheinen, sofern ihnen ein negierter Hauptsatz vorausgeht. 
(52) Es gab nie einen Maler Heidemann, dem Hitler Modell gesessen hätte. (FOCUS 1993, zitiert in DUDEN 2009: 522)

(53) Ich habe in den sechs Jahren noch nicht erlebt, dass mir jemand offen Einblick ins Innerste gegeben hätte. (ZEIT 1996, zitiert in ebd.: 522)

\section{Höflichkeit: Der Konjunktiv II in höflichen Aufforderungen, Feststellungen und Bitten}

Mit dem Konjunktiv II kann man außerdem höflich um etwas bitten, direkte Aufforderungen umgehen sowie vorsichtige und zurückhaltende Feststellungen tätigen. In diesen Fällen ist der Konjunktiv II nicht durch den Konjunktiv I austauschbar (vgl. DUDEN 2009: 521). Ein Ersatz des Konjunktivs der Höflichkeit durch den Indikativ ist zwar rein theoretisch möglich, da sich der Sinn eines Satzes nicht wesentlich ändert. Verwendet der Sprecher allerdings den Indikativ, so entfällt die subjektive Distanzierung, die der Konjunktiv II ermöglicht. Eine Bitte beispielsweise wirkt schroffer (vgl. DUDEN 2009: 521, HEIDOLPH et al. 1981: 533). Durch die Verwendung des höflichen Konjunktivs macht sich der Sprecher die irreale Unterstellung, die mit dem Konjunktiv verbunden ist, zu Nutze und verschafft sich auf diese Weise mehr Handlungsspielraum (vgl. DUDEN 2009: 521).

\section{Konjunktiv in Verbindung von Modalverben}

Auch Modalverben können in den Dienst der Modalität gestellt werden. Man verwendet sie zum Ausdruck von erfüllbaren Wünschen, Aufforderungen unterschiedlichen Verbindlichkeitsgrades und Vermutungen unterschiedlichen Gewissheitsgrades, Behauptungen fremder Personen, folgenlos bleibenden Sachverhalten und nicht mehr Realisierbarem (vgl. JUNG 1984: 231f.). Wie die folgenden Beispielsätze zeigen, können Modalverben mit oder ohne Konjunktiv auftreten. Allerdings gilt anzumerken, dass bei einem Ersatz des Konjunktivs durch den Indikativ die subjektive Distanzierung entfällt (vgl. HEIDOLPH et al. 1981: 533)

(54) erfüllbare Wünsche: Ich möchte schwimmen gehen. Du könntest mir helfen.

(55) Aufforderungen: Du solltest / müsstest für die Schule lernen.

(56) Vermutungen: Diese Rechnung könnte / dürfte / müsste richtig sein.

(57) Behauptungen: Tom ist nicht in der Schule. Er soll angeblich krank sein. 
Geith, V. - Konjunktiv als Mittel der Bedeutungsschattierung

(58) folgenlos bleibende Sachverhalte: Mag das auch aussichtslos erscheinen, ich kämpfe weiter.

(59) nicht mehr Realisierbares: Du hättest mich fragen können.

\section{Die Darstellung des Konjunktivs in Lern- und Übungsgrammatiken}

In Handbüchern, Grammatiken und linguistischen Lexika werden viele, sehr unterschiedliche Kontexte genannt, in denen Konjunktiv I und II zum Einsatz kommen. Die beiden vorangegangenen Abschnitte sollten einerseits eine Übersicht über diese Vielzahl an Verwendungsmöglichkeiten bieten. Andererseits sollten sie dafür sensibilisieren, dass es gerade mit wachsender Kenntnis der Einsatzmöglichkeiten schwieriger wird, sich einen Überblick zu verschaffen bzw. diesen zu behalten, gerade wenn man sich neu in die Thematik einarbeitet. Dies mag für angehende DaFLehrer/innen gelten sowie für Nicht-Muttersprachler. Aufgrund der Vielfältigkeit und der Unterschiedlichkeit der Einsatzmöglichkeiten kann es für NichtMuttersprachler/innen schwer sein, die Bedeutung des Konjunktivs zu greifen, und für Lehrpersonen mag es schwierig erscheinen, diese zu vermitteln.

Gerade vor diesem Hintergrund kommt Lern- und Übungsgrammatiken eine zentrale Rolle im DaF-Unterricht zu. Daher untersucht der kommende Abschnitt, wie ausgewählte Lern- und Übungsgrammatiken den Konjunktiv darstellen. Die Grammatiken wurden dabei nach folgenden Gesichtspunkten verglichen:

a) Welche Beispiele werden für die Semantik des Konjunktivs I und die Semantik des Konjunktivs II gegeben?

b) Gibt es eine deutliche Abgrenzung des Konjunktivs vom Indikativ oder werden lediglich Sätze im Indikativ einigen Sätzen im Konjunktiv gegenübergestellt? Ist diese Abgrenzung umfassend oder bezieht sie sich nur auf einige Teilbereiche?

c) Wird der Konjunktiv als ein Mittel der Modalität beschrieben? Bzw. wird beschrieben, was Modalität ist?

Die Antworten auf diese Fragen werden im Folgenden beschrieben. Im Anhang befinden sich zudem Tabellen, die die Ergebnisse der Untersuchung überblicksartig darstellen. 
Semantik des Konjunktivs I in den Lern- und Übungsgrammatiken

Mit Ausnahme der Grammatik für Anfänger von BuschA / SzITA (2010) erwähnen alle Grammatiken, dass der Konjunktiv I in der indirekten Rede verwendet werden kann. Allerdings spezifizieren nur einige, welche Konsequenzen sich daraus ergeben. Folgende Spezifizierungen wurden bei den Autoren gefunden:

a) Wiedergabe von Meinungen und Äußerungen (vgl. BusCHA / SzITA 2011: 90, Dies. 2013: 77, DREYER / SCHMITT 2000: 257, Dies. 2009: 278, RUG / TOMASZEWSKI 2009: 103)

b) Distanz zur Meinung einer anderen Person (vgl. STEIN-BASSLER 2008: 122, FANDRYCH 2012: 176, RUG / TOMASZEWSKI 2009: 109)

c) Verdeutlichung: Bezweiflung der Richtigkeit der Aussage (vgl. STEIN-BASSLER 2008: 124)

d) Wiedergabe einer Rede aus der Sicht des Sprechers (vgl. HaLl / ScHEINER 2006: 119)

e) Verdeutlichung: keine Garantie für die Richtigkeit der Aussage （vgl. ClameR et al. 2006: 105)

f) Objektivierung einer Aussage (vgl. HALl / ScHEINER 2008: 120, BuSCHA / SzITA 2011: 90, DREYER / SCHMITT 2000: 275, Dies. 2009: 298, RUG / TOMASZEWSKI 2009: 109)

g) Verdeutlichung: Verantwortung für das Gesagte liegt bei einer anderen Person (RUG / TOMASZEWSKI 2009: 109, FANDRYCH 2012: 176)

h) Verkürzung einer Aussage (vgl. BuschA / SzITA 2011: 90, DREYER / SCHMITT 2000: 275, Dies. 2009: 298)

Die anderen Verwendungen des Konjunktivs I werden nur von einigen Lern- und Übungsgrammatiken erwähnt. Beispielsätze wurden gesammelt und entsprechend der im Abschnitt 5 aufgeführten Unterteilung hier aufgelistet.

Der Volitiv bei direkt geäußerten Wünschen und Bitten kommt in drei Grammatiken vor. 
Geith, V. - Konjunktiv als Mittel der Bedeutungsschattierung

(60) Möge sie lange leben! Sie lebe hoch! Edel sei der Mensch, hilfreich und gut! (HALL / SCHEINER 2006: 118)

(61) Hoch lebe das Brautpaar! Mögest du dich [...] guter Gesundheit erfreuen! Gott behüte dich! Gott sei Dank! (CLAMER et al. 2006: 107)

(62) Es lebe der König! Er ruhe in Frieden. (BUSCHA / SZITA 2013: 77)

Zur Äußerung von indirekten Wünschen und Bitten in Verbindung mit Finalsätzen lässt sich nur ein Beispiel finden:

(63) Der Arzt impfte sie, damit sie im Ausland nicht krank werde / wird. (HALL / SCHEINER 2006: 119)

Aufforderungen in der 1. und 3. Person Plural gibt es nur in einer Grammatik:

(64) Seien wir vernünftig! Seien Sie so gut, mir beim Tragen zu helfen. (HALL / SCHEINER 2006: 118)

Den Volitiv bei Aufforderungen in (mathematischen, physikalischen) Fachtexten findet man zweimal:

(65) Gegeben sei eine Gerade [...]. (HALL / SCHEINER 2006: 119)

(66) Gegeben sei ein gleichseitiges Dreieck [...]. (CLAMER et al. 2006: 108)

Der Volitiv bei Aufforderungen in Verbindung mit man wird in folgenden Beispielen erwähnt:

(67) Man nehme morgens und abends jeweils eine Tablette. (HALL / SCHEINER 2006: 118)

(68) Nach der Montage des Getriebes führe man einen Probelauf [...] durch. (CLAMER et al. 2006: 108)

Der Konjunktiv I in formelhaften Konzessivsätzen bei Einräumungen und Zugeständnissen findet Eingang in:

(69) Was auch immer geschehe, ich halte zu dir. (HALL / SCHEINER 2006: 119)

(70) Ich komme sicher mit dem Auto, es sei denn, es springt mal wieder nicht an. Komme, was (da) wolle. (BUSCHA / SZITA 2013: 76)

Beispielsätze für die Umschreibung des Autorenplurals findet man in folgenden Sätzen.

(71) In diesem Zusammenhang sei daran erinnert. (HALL / SCHEINER 2006: 119)

Pandaemonium, São Paulo, v. 19, n. 29, nov.-dez. 2016, p. 53-94 
Geith, V. - Konjunktiv als Mittel der Bedeutungsschattierung

(72) Es sei hier noch einmal auf die Möglichkeit hingewiesen. (CLAMER et al. 2006: 108)

Semantik des Konjunktivs II in den Lern- und Übungsgrammatiken

Alle Grammatiken erwähnen, dass man den Konjunktiv II in Konditionalsätzen verwenden kann, um hypothetische und irreale Bedingungen zu beschreiben bzw. um theoretisch mögliche und nicht mehr mögliche Handlungsalternativen darzubieten. Folgende Beispiele kann man finden:

(73) Wenn er käme, würde ich mich freuen. (STEIN-BASSLER 2008: 121)

(74) Wenn der [...] Fahrer nicht [...] herumgerissen hätte, wären beide Autos frontal zusammengestoßen. (HALL / SCHEINER 2006: 94)

(75) Er wäre rechtzeitig gekommen, wenn er ein Taxi genommen hätte. (CLAMER et al. 2006: 96)

(76) Wenn Claudia Geld hätte, würde sie sich ein Auto kaufen. (BUSCHA / SZITA 2010: 53; Dies. 2011: 87)

(77) Wenn ich Astronaut wäre, könnte ich zum Mond fliegen. (BUSCHA / SZITA 2013: 67)

(78) Wenn du mit dem ICE gefahren wärest, wärest du weniger gestresst in Hamburg angekommen. (RUG / TOMASZEWSKI 2009: 55)

(79) Wenn ich Zeit hätte, würde ich sehr gerne kommen. (REIMANN 2000: 66)

(80) Wenn ich den Job bekommen würde, dann hätte ich mehr Geld. (HERING et al. 2009: 134)

(81) Wenn Sie Geschichte studiert hätten, dann hätten Sie sofort gewusst, worum es dabei geht. (FANDRYCH 2012: 170)

(82) Wenn ich Zeit hätte, käme ich zu dir. (DREYER / SCHMITT 2000: 263)

(83) Wenn wir damals genug gespart hätten, (so / dann) hätten wir das Haus gekauft. (DREYER / SCHMITT 2009: 285)

Ebenfalls bei allen Grammatiken vorhanden ist die Darstellung von erfüllbaren und unerfüllbaren Wünschen im Konjunktiv II:

(84) Wenn ich ihn doch sehen könnte! (STEIN-BASSLER 2008: 119)

(85) Hätte der Autofahrer doch nicht überholt! (HALL / SCHEINER 2006: 94)

(86) Hätte ich doch mehr Grammatik gelernt! (CLAMER et al. 2006: 96)

(87) Sie würde gern in den Urlaub fahren, aber sie hat keine Zeit. (BUSCHA / SZITA 2010: 53)

(88) Er wäre gern wieder gesund. Wäre er doch wieder gesund! (BUSCHA / SZITA 2011: 87)

(89) Ich wäre gern Astronaut. Wäre ich doch nur Astronaut! (BUSCHA / SZITA 2013: 67)

(90) Wenn ich doch im Denken nicht so langsam wäre! (RUG / TOMASZEWSKI 2009: 55)

(91) Wenn ich doch mein Geld mitgenommen hätte! Hätte ich doch mein Geld mitgenommen! (REIMANN 2000: 67)

(92) Wenn der Typ doch endlich verschwinden würde! (HERING et al. 2009: 136)

(93) Hätte er mich doch nur hören können! (FANDRYCH 2012: 171) 
Geith, V. - Konjunktiv als Mittel der Bedeutungsschattierung

(94) Wenn sie nur (oder: doch nur) mitgefahren wären! Wären sie nur (oder: doch nur) mitgefahren! (DREYER / SCHMITT 2000: 261, Dies. 2009: 283)

Bis auf die Grammatik von BuSCHA / SzITA (2010) für Anfänger erwähnen alle Grammatiken Komparativsätze. Folgende Beispiele für irreale Vergleiche sind zu finden:

(95) Er tut so, als ob er alles wüsste. (STEIN-BASSLER 2008: 111)

(96) Es sieht so aus, als ob der Beifahrer einen Schock erlitten hätte (habe). (HALL / SCHEINER 2006: 95)

(97) Er fährt, als ob er ein Rennfahrer wäre! (CLAMER et al. 2006: 96)

(98) Frau Müller tut so, als wären unsere Probleme völlig unwichtig. (BUSCHA / SZITA 2011: 89)

(99) Er tut so, als ob er mich nicht sehen würde. (BUSCHA / SZITA 2013: 67)

(100) Die Gazelle argumentiert so, als ob sie wie ein Löwe wäre. (RUG / TOMASZEWSKI 2009: 56)

(101) Er ist faul, aber er tut so, als ob er arbeiten würde. (REIMANN 2000: 67)

(102) Du siehst so aus, als ob du gerade ein Gespenst gesehen hättest. (HERING et al. 2009: 138)

(103) Als hätte er nichts zu tun. (FANDRYCH 2009: 173)

(104) Sie schaut mich an, als ob sie mich nicht verstünde. (DREYER / SCHMITT 2000: 266, Dies. 2009: 288)

Fast durchgängig wird auch der Konjunktiv der Höflichkeit erwähnt. Die Lern- und Übungsgrammatiken sind diesbezüglich ausführlicher als linguistische Lexika, Handbücher und Grammatiken, denn die Liste ist lang: Der Konjunktiv II wird gebraucht, um höfliche Bitten, Fraugen, Aufforderungen, Ratschläge, Empfehlungen, Vorschläge, Meinungsäußerung oder nachträgliche Kritik zu äußern. Mit Ausnahme von STEIN-BASSLER, die den Konjunktiv der Höflichkeit ebenfalls erwähnt, aber kein Beispiel gibt, weist jede Grammatik einen Beispielsatz auf.

(105) An Ihrer Stelle würde ich nicht an der Unfallstelle bleiben. (HALL / SCHEINER 2006: 95)

(106) Könnte ich bitte noch einen Kaffee haben? (CLAMER et al. 2006: 96)

(107) Könnte ich bitte mit Herrn Müller sprechen? (BUSCHA / SZITA 2010: 51)

(108) Ich würde das (an deiner Stelle) noch einmal überlegen. (BUSCHA / SZITA 2011: 81)

(109) Du hättest vorher fragen sollen / müssen. (BUSCHA / SZITA 2013: 67)

(110) Würden Sie bitte bei dieser Aufgabe lächeln? (RUG / TOMASZEWSKI 2009: 53)

(111) An deiner Stelle würde ich mir vor der langen Fahrt noch etwas zu essen kaufen. (REIMANN 2000: 67)

(112) Könnten Sie mir sagen, ob Sie Richtung Alex fahren? (FANDRYCH 2009: 175)

(113) Wären Sie so freundlich, mir zu helfen? (DREYER / SCHMITT 2000: 269, Dies. 2009: 292) 
Geith, V. - Konjunktiv als Mittel der Bedeutungsschattierung

Ebenso gestaltet es sich beim Konjunktiv II als Ersatz für Konjunktiv I in der indirekten Rede. Diese Verwendungsweise wird nahezu von jeder Grammatik erwähnt. STEINBASSLER erwähnt auch diese Verwendungsweise (2008: 111, 122), verbleibt aber erneut ohne Beispiel.

(114) Er behauptet, dass ihn die Sonne geblendet hätte (HALL / SCHEINER 2006: 95)

(115) Sie sagten, dass sie abends nie spazieren ginge und dass sie auch keinen Hund hätten. (CLAMER et al. 2006: 96)

(116) Susi sagte, sie müsste die Zahlen noch einmal überprüfen. (BUSCHA / SZITA 2011: 90)

(117) Er sagte, er hätte sein Heft zu Hause vergessen. (BUSCHA / SZITA 2013: 67)

(118) Er hat mir gesagt, die Heinzelmännchen kämen heute Abend und würden uns helfen. (RUG / TOMASZEWSKI 2009: 104)

(119) Die Oppositionsparteien betonten, sie hätten ein besseres Steuerkonzept. (HERING et al. 2009: 140)

(120) Er hat mir vorhin erzählt, dass er schon seit 3 Monaten ein Praktikum suchen würde. (FANDRYCH 2012: 176)

Sechs der verglichenen Grammatiken erwähnen den Konjunktiv II in Zusammenhang mit Modalverben. Sie verweisen darauf, dass er dann eine Vermutung oder eine Möglichkeit ausdrücken kann. Hier gilt es anzumerken, dass die Lern- und Übungsgrammatiken vereinfacht darstellen, denn auch ohne den Konjunktiv II können manche Modalverben eine Vermutung ausdrücken, etwa in dem Satz: Er mag krank sein. Die Vermutung ist also weniger an das Kriterium Konjunktiv, sondern mehr an das Modalverb gebunden.

(121) Er dürfte langsam fertig sein. (STEIN-BASSLER 2008: 111)

(122) Der rasante Autofahrer wäre vielleicht ein guter Rennfahrer. (HALL / SCHEINER 2006: 95)

(123) Vera dürfte inzwischen mit dem Studium fertig sein. (CLAMER et al. 2006: 96)

(124) Das dürfte schon klappen. (FANDRYCH 2012: 174)

(125) Zum Einkaufen dürfte es jetzt zu spät sein. (DREYER / SCHMITT 2000: 269)

(126) Für meine Verabredung dürfte es jetzt zu spät sein. (DREYER / SCHMITT 2009: 292)

Dass der Konjunktiv II zum Ausdruck von irrealen Folgen verwendet werden kann, wird ebenfalls bei fünf der untersuchten Grammatiken beschrieben.

(127) Es ist zu kalt, als dass man schwimmen könnte. (STEIN-BASSLER 2008: 111)

(128) Er ist zu verwirrt, als dass er die Fragen der Polizisten beantworten könnte (kann). (HALL / SCHEINER 2006: 95)

(129) Die Fenster sind noch zu sauber, als dass du sie schon wieder putzen müsstest. (CLAMER et al. 2006: 96)

(130) Das Buch ist zu langweilig, als dass man wach bleiben könnte. (HERING et al. 2009: 136) 
Geith, V. - Konjunktiv als Mittel der Bedeutungsschattierung

(131) Es ist nicht so, dass es den Zuschauer nicht stören würde [...]. (FANDRYCH 2009: 173)

(132) Es ist zu spät, als dass wir noch bei ihm anrufen könnten. (DREYER / SCHMITT 2000: 267)

(133) Als Rentner habe ich so viel Zeit, dass ich das ganze Jahr verreisen könnte. (DREYER / SCHMITT 2009: 290)

Konsekutivsätze mit Konjunktiv II werden nur bei zwei Übungsgrammatiken erwähnt.

(134) Auch wenn ich ein schnelles Auto hätte, würde ich nicht so rasen. (HALL / SCHEINER 2006: 95)

(135) Auch wenn ich die Möglichkeit gehabt hätte, etwas in meinem Leben zu ändern, so hätte ich doch nichts [...] geändert. (FANDRYCH 2009: 171)

Relativsätze nach negierter Feststellung sind in drei Grammatiken zu finden.

(136) Es gibt keinen Autofahrer, der vor Unfällen sicher wäre. (HALL / SCHEINER 2006: 95)

(137) Ich kenne keinen anderen Arzt, der dir besser helfen könnte. (DREYER / SCHMITT 2000: 270, Dies. 2009: 292)

Die im Folgenden aufgelisteten Verwendungsweisen wurden nicht in den linguistischen Lexika, Handbüchern und Grammatiken beschrieben. Und auch die Lern- und Übungsgrammatiken stellen sie nur vereinzelt dar. Hierzu gehören:

a) verpasste Gelegenheiten bzw. Ereignisse, die wider Erwarten nicht eingetreten sind; diese Gelegenheiten / Ereignisse treten mit fast oder beinah(e) auf.

(138) Fast hätte es einen frontalen Zusammenstoß gegeben. (HALL / SCHEINER 2006: 95)

(139) Fast hätte er einen Oskar bekommen. (BUSCHA / SZITA 2011: 89)

(140) Fast / Beinahe hätte ich fünf Millionen Euro gewonnen. (BUSCHA / SzITA 2013: 67)

(141) Beinah(e) wäre das ganze Haus abgebrannt! (DREYER / SCHMITT 2000: 269)

(142) Beinah(e) wäre ich nicht mehr rechtzeitig gekommen! (DREYER / SCHMITT 2000: 292)

(143) Fast hätte ich den Bus nicht mehr erreicht. (DREYER / SCHMITT 2000: 269, Dies. 2009: 292)

b) Sätze mit sonst oder andernfalls

(144) Dieser Fahrer hat schnell reagiert, sonst hätte es einen Unfall gegeben. (HALL / SCHEINER 2006: 95)

(145) Ehepartner müssen / sollten Rücksicht aufeinander nehmen, andernfalls gäbe / gibt es ständig Streit. (HALL / SCHEINER 2006: 105)

(146) Er musste ein Taxi nehmen, sonst wäre er zu spät gekommen. (DREYER / SCHMITT 2000: 265, Dies. 2009: 288) 
Geith, V. - Konjunktiv als Mittel der Bedeutungsschattierung

c) zweifelnde Fragen bzw. Erstaunen, mit dem man die Welt, so wie sie ist, in Frage stellt; oft treten sie mit den Verben sollen und können auf.

(147) Ich hätte nie gedacht, dass die Polizei so schnell am Unfallort ist. Ob ich auch so schnell reagiert hätte? (HALL / SCHEINER 2006: 95)

(148) Würdest du das (an meiner Stelle) tun? (RUG / TOMASZEWSKI 2009: 55)

(149) Sollte es wirklich schon so spät sein? (DREYER / SCHMITT 2000: 269, Dies. 2009: 292)

(150) Würdest du mir tatsächlich Geld leihen? (DREYER / SCHMITT 2000: 269)

(151) Könnte die Frist tatsächlich schon abgelaufen sein? (DREYER / SCHMITT 2009: 292)

d) abschließende Feststellungen, mit denen man ausdrückt, dass ein Teil des Sachverhalts beendet ist

(152) Das hätten wir geschafft! (HALL / SCHEINER 2006: 95)

(153) So, das wär's für heute! (Morgen geht's weiter.) (DREYER / SCHMITT 2000: 270, Dies. 2009: 292)

e) Verbindung mit Verben des Sagens wie annehmen, glauben, denken, meinen, wundern, sagen auftreten; sie werden bei DREYER / SCHMITT mit „Unsicherheiten“ betitelt (2000: 270, 2009: 292), während sie bei RUG / TOMASZEWSKI unter „Redewendungen“ (2009: 56) aufgeführt werden.

(154) Ich glaube, dass ich ihm in dieser Lage auch nicht helfen könnte. (DREYER / SCHMITT 2000: 270, Dies. 2009: 292)

(155) Man sollte meinen (denken, glauben), wir wären hier einem zivilisierten Land. Es wäre schön (toll, prima), wenn wir uns mal wieder sehen könnten. (RUG / TOMASZEWSKI 2009: 56)

f) RUG und TOMASZEWSKI schließen in ihrer Liste der Sätze auch ein Beispiel ein, in dem sich der Sprecher die Welt, so wie sie ist, anders vorstellt

(156) Ich hätte alles besser gewusst und das meiste auch besser gemacht. (RUG / TOMASZEWSKI 2009: 55)

g) Und bei DrEYER und SchmitT findet man noch Beispiele für die Verwendung des Konjunktivs zur Unterscheidung von Realität und Irrealität.

(157) Ich hätte dich besucht, aber ich hatte deine Adresse nicht. (DREYER / SCHMITT 2000: 269) 
(158) Ich hätte dich besucht, aber ich hatte leider einen dringenden Termin. (DREYER / SCHMITT 2009: 292)

(159) Der Bus ist noch nicht da; dabei hätte er schon vor zehn Minuten kommen müssen. (DREYER / SCHMITT 2000: 269, Dies. 2009: 292)

Deutliche, umfassende und allgemeine Abgrenzung von Konjunktiv zu Indikativ

Hier wurde untersucht, ob eine deutliche und umfassende Abgrenzung von dem unmarkierten Modus Indikativ zu dem markierten Modus Konjunktiv vorgenommen wurde. Gesucht wurde etwa nach Beschreibungen, die darauf hinweisen, dass der Indikativ die bestehende Wirklichkeit bezeichnet und der Konjunktiv sich auf eine gedachte bzw. hypothetische Welt bezieht. Des Weiteren wurde analysiert, ob die Abgrenzung allgemein vollzogen wurde, oder nur ausschnittsweise anhand einiger Beispiele geschah. Bezüglich dieses Aspekts lassen sich die Grammatiken in drei Gruppen aufteilen.

a) Die Grammatiken der ersten Gruppe erklären den Unterschied von Indikativ und Konjunktiv deutlich, umfassend und allgemein. Sie sind jedoch in der Unterzahl. $\mathrm{Zu}$ dieser Gruppe gehören: DreYer / SchmitT (2000: 257), Buscha / SzITA (2013: 65), Stein-Bassler (2008: 110) und Hall / ScheINER (2006: 96). Die Tabelle 2 im Anhang zeigt, mit welchen Worten die Grammatiken die Abgrenzung vollziehen.

b) Die zweite Gruppe begnügt sich mit einer Gegenüberstellung von Sätzen im Indikativ und im Konjunktiv. Indikativische und konjunktivische Sätze bekommen dann zwar zumeist einen Zusatz, wie z.B. Realität und Irrealität. Es folgen aber keine weiteren Erklärungen, die den Unterschied der beiden Modi verdeutlichen würden. $\mathrm{Zu}$ bemerken ist außerdem, dass meistens nur in einem Teilbereich Sätze im Indikativ und im Konjunktiv gegenübergestellt werden. Eine Kontrastierung findet nicht bei allen Kontexten statt, in denen der Konjunktiv verwendet werden kann. In dieser Gruppe sind: FANDRYCH (2012: 170), RUG / TOMASZEWSKI (2009: 103), BuSCHA / SZITA (2010: 51, 54) und HERING et al. (2009: 134).

c) Bei den übrigen Grammatiken schließlich findet man weder eine deutliche Erklärung der Modi, noch eine Gegenüberstellung von indikativischen und 
konjunktivischen Sätzen. Interessanterweise ist die Grammatik von DREYER / SCHMITT aus dem Jahr 2009 weniger deutlich, was eine Abgrenzung von Konjunktiv und Indikativ angeht.

\section{Konjunktiv als Mittel der Modalität}

Hier wurde untersucht, ob die Grammatiken den Modus als eine Aussagweise des Verbes darstellen und ob sie darauf hinweisen, dass der Konjunktiv die Qualität der Aussage verändert, dass er einen Sachverhalt als nicht wirklich, sondern möglich, unsicher, vermutet etc. präsentiert. Es wurde nach Beschreibungen gesucht, die darauf verweisen, dass der Konjunktiv den Wahrheits- bzw. den Wirklichkeitsbezug einer Aussage bestimmt und dadurch das Verb ,färbt'. Als Ergebnis der Untersuchung lässt sich feststellen, dass nur bei einer Grammatik eine solche Darstellung vorhanden ist. Bei Dreyer / SCHMitT (2000: 257) wird der Modus als Aussageweise definiert und beschrieben, dass mit dem Modus Indikativ eine Aussage als wirklich oder wirklich geglaubt hingestellt werden kann. Der Konjunktiv wird als eine andere Aussageweise, als ein anderer Modus, präsentiert, mit dem man die Rede einer anderen Person wiedergibt oder etwas Irreales, nicht Wirkliches darstellt.

BusCHA und SzITA definieren Indikativ, Imperativ und Konjunktiv zwar als Aussageweisen des Verbes. Aber es wird keine weitere Erklärung gegeben, was unter Aussageweise zu verstehen ist (vgl. 2013: 65). Bei FANDRYCH gibt es ein extra Kapitel über Modalität (vgl. 2012: 158-175). In diesem werden beispielsweise Modalverben als Ausdruck von Bedingungen des Handels präsentiert. Der Konjunktiv wird auch in diesem Kapitel aufgelistet. Es wird jedoch nicht erklärt, welche Konsequenzen sich daraus ergeben.

Bei allen anderen Grammatiken gibt es keine weiteren Erklärungen zum Konjunktiv als einem Mittel der Modalität. Der Begriff Modus wird in den meisten Grammatiken nicht aufgeführt. Interessanterweise ist dies auch der Fall bei der überarbeiteten und aktualisierten Auflage von DREYER / SCHMITT aus dem Jahr 2009. 


\section{Abschließende Bemerkungen}

Diese Beobachtungen werfen eine Reihe von Fragen auf. Warum grenzen die meisten Grammatiken Konjunktiv und Indikativ nicht systematisch und umfassend voneinander ab? Genügt eine bloße Gegenüberstellung von indikativischen und konjunktivischen Sätzen? Wenn dem so wäre, warum wird diese Gegenüberstellung nur in einigen Teilbereichen vorgenommen? Warum beschreibt die Mehrzahl der Grammatiken den Konjunktiv nicht explizit als Mittel der Modalität? Und warum führen die meisten Grammatiken den Begriff Modus nicht einmal auf? Der Großteil der Grammatiken begnügt sich mit einer Auflistung der verschiedenen Verwendungsweisen der Konjunktive. Doch kann dadurch ein umfassendes Verständnis des Konjunktivs gewährleistet werden? Kann durch die Darstellung einer Vielzahl an Satzstrukturen, in denen der Konjunktivgebrauch möglich ist, aufgezeigt werden, was der Konjunktiv leistet?

Die Modalität als Mittel der „Bedeutungsschattierung“ (JUNG 1984: 233) ist das verbindende Element für jeden Konjunktivgebrauch und das sollte den Lernern deutlich vermittelt werden. Begnügt man sich nämlich mit der bloßen Darstellung von Sätzen, in denen Konjunktiv vorkommen kann, greift dies zu kurz. Beschreibt man beispielsweise, dass Konjunktiv II in irrealen Wunschsätzen verwendet werden kann, erklärt dies nur hinreichend die Bedeutung des Konjunktivs. Denn es wird nicht deutlich, warum in diesen Sätzen der Konjunktiv auch durch den Indikativ ersetzt werden kann, und welche Konsequenzen sich durch einen Austausch ergeben. Es wird nicht deutlich, dass ein Wunsch mit Konjunktiv weniger starken Wirklichkeitscharakter hat, und er mit dem in Hinblick auf eine Realisierung des Sachverhaltes - zuversichtlicheren Wunsch mit Indikativ kontrastiert.

Es gibt zwar einige wenige irreale Nebensätze, die auch ohne Konjunktiv eine irreale Deutung suggerieren oder sogar fordern. Diese Irrealität wird in Komparativätzen mit als wenn, wie wenn und als ob durch die Subjunktoren selbst gewährleistet. Dasselbe gilt für Konsekutivsätze mit als dass, ohne dass und dass (nach $z u$ oder nicht so) und für Relativ- oder andere Nebensätze, die nach einem negierten Hauptsatz auftreten.

Mit Ausnahme von diesen Sätzen macht es einen Unterschied, ob ein Sprecher Indikativ oder Konjunktiv wählt. Die Wahl des Konjunktivs zeigt, dass der Sprecher 
dem Gesagten eine eingeschränkte Geltung zuspricht und dass er das Gesagte nicht als gesetzt verstanden wissen will. Die Wahl des Konjunktivs steht der Wahl des Indikativs entgegen, mit der ein Wirklichkeitsbezug hergestellt wird. Der im Konjunktiv stehende Satz impliziert eine Distanz zu diesem Wirklichkeitsbezug, er verweist auf Vorsicht und Zurückhaltung des Sprechers. Bei einer Rückkehr zum Indikativ wird die eingeschränkte Geltung des Gesagten aufgehoben und ein so umgewandelter Satz kann gegebenenfalls sogar das Gegenteil von dem zuvor Ausgedrückten darstellen. Genau das sollte den Deutschlernern/innen auch vermittelt werden. Für niedrigere Lernniveaus ist diese differenzierte und abstrakte Darstellung der Bedeutungstiefen der Modi unter Umständen nicht angebracht und nicht nötig. Aber gerade für höhere Lernniveaus auf C-Niveau kann die Vermittlung des Konjunktivs als einem Mittel der Modalität hilfreich sein. Denn je weiter Nicht-Muttersprachler/innen in ihrem Lernprozess voranschreiten, desto mehr Verwendungsmöglichkeiten lernen sie kennen, desto breiter und unübersichtlicher wird das Spektrum der Einsatzmöglichkeiten. Je höher das Lernniveau ist, desto mehr steigt das Vermögen, abstraktere Grammatikerklärungen, die losgelöst von konkreten Satzstrukturen, spezifischen Subjunktoren, redeeinleitenden Verben etc. gegeben werden, nachzuvollziehen. Die Vermittlung der Bedeutung des Konjunktivs als einem Mittel der „Bedeutungsschattierung“ (JUNG 1984: 233) ist gerade auch deshalb wichtig, weil der Konjunktiv nicht immer mit eindeutig erkennbaren Kennzeichen einhergeht: Er kann ohne spezifische Subjunktoren, redeeinleitende Verben usw. auftreten. Denkt man an verkappte Konditionalsatzgefüge, irreale Wunschsätze mit Spitzenstellung des Verbes oder satzeinleitende Wendungen wie angenommen, so wird das deutlich. Gerade deswegen ist es wichtig, darauf zu verweisen, dass solche Sätze im Konjunktiv einen vorgestellten, nicht faktischen Hintergrund schaffen.

\section{Schlussbetrachtung}

Aus den oben dargestellten Besonderheiten des deutschen Konjunktivs wird ersichtlich, warum es Deutschlernern/innen schwer fallen kann, den Konjunktiv zu verstehen und anzuwenden. Das Verständnis des Konjunktivs wird aufgrund des Formensynkretismus erschwert. Viele Formen des Konjunktivs I gleichen dem Indikativ, viele Formen des Konjunktivs II sind identisch mit dem Präteritum. Beim Lesen von Texten können sich 
daher Verständnisschwierigkeiten ergeben, wenn zweideutige Konjunktivformen nicht durch würde-Formen ersetzt wurden, weil aus dem Kontext ableitbar wäre, dass es sich um Konjunktiv handelt. Eine weitere Schwierigkeit kann aus der fehlenden temporalen Differenzierung resultieren. Neben den Problemen bei der Rezeption, kann es auch zu Schwierigkeiten bei der Anwendung kommen. Denn Konjunktiv I und Konjunktiv II werden in zahlreichen Kontexten und unterschiedlichen Satzstrukturen verwendet. Daher ist es auch verständlich, wenn eine gewisse Unsicherheit hinsichtlich dessen besteht, was der Konjunktiv leistet. Sucht man in dieser Unsicherheit Rat bei Lern- und Übungsgrammatiken, so wird man unter Umständen enttäuscht.

Viele dieser Grammatiken zeigen, wie der Konjunktiv gebildet wird und erklären detailliert die verschiedenen Verwendungsweisen. Sie beschreiben ausführlicher als Handbücher, Grammatiken und linguistische Lexika, wann eine würde-Form eine uneindeutige Konjunktivform ersetzt. Doch die Mehrheit stellt nicht deutlich dar, was alle Konjunktivformen eint. Viel eher wird in einer Fülle von Beispielsätzen beschrieben, in welchen Situationen der Konjunktiv auftreten kann. Angesichts der vielfältigen Verwendungsweisen des Konjunktivs kann es den Deutschlernern/innen eine Hilfe sein, wenn man verstärkt darauf hinweist, dass der Konjunktiv ein Mittel der Modalität, ein Mittel zu „Färbung“ (HOMBERGER 2000: 342) einer Aussage, ist. Er verändert den Bedeutungsgehalt einer Aussage. Gerade deswegen ist es wichtig, den Konjunktiv systematisch und explizit vom Indikativ abzugrenzen und darzustellen, dass der Indikativ der unmarkierte Modus ist, der sich um die bestehende Wirklichkeit dreht, und dass der Konjunktiv als der markierte Modus angibt, dass der Inhalt einer Aussage nicht voraussetzungslos, sondern nur unter bestimmten Bedingungen gilt. Als Deutschlerner/in sollte man nicht nur wissen, in welchen konkreten Satzgefügen und nach welchen Subjunktoren man den Konjunktiv verwenden kann. Man sollte auch seine Bedeutungstiefe greifen können und verstehen, dass der Konjunktiv die Qualität der Aussage verändert und die Macht hat, einen Sachverhalt als nicht wirklich, sondern möglich, vermutet, unsicher, vermittelt etc. darzustellen. Daher lässt sich abschließend Folgendes sagen: Gerade weil es schwer ist, die Verwendungsmöglichkeiten des Konjunktivs auf einen Nenner zu bringen, sollte mit fortschreitendem Niveau der Lerner/innen der Konjunktiv als Mittel der „Bedeutungsschattierung“ (JUNG 1984: 233) vermittelt werden. Und wenn Lern- und Übungsgrammatiken, mit denen man im Unterricht arbeitet, dieses verbindende 
Element nicht explizit erwähnen, erscheint es umso angebrachter, dies im Klassenraum $\mathrm{zu}$ thematisieren.

\section{Anhang}

Die folgenden zwei Tabellen zeigen, welche Verwendungsweisen des Konjunktivs die einzelnen Lern- und Übungsgrammatiken erwähnen. Die Seitenzahlen verweisen dabei auf zweierlei: Auf der einen Seite geben sie beim Indirektheitskonjunktiv an, auf welchen Seiten die Grammatiken Spezifizierungen vornehmen und erklären, wozu der Konjunktiv der indirekten Rede (iR) dient. Gibt es keine Spezifizierung, wurde dies mit Spez. gekennzeichnet. Auf der anderen Seite verweisen die restlichen Seitenzahlen darauf, auf welcher Seite ein Beispiel für die jeweilige Verwendungsweise zu finden ist. Wurde die Verwendungsweise ohne konkretes Beispiel erwähnt, wurde dies mit Bsp. ausgedrückt.

TABELLE 1a: Semantik von Konjunktiv I und II

\begin{tabular}{|c|c|c|c|c|c|c|}
\hline & $\begin{array}{l}\text { STEIN- } \\
\text { BASSLER } \\
(2008)\end{array}$ & $\begin{array}{l}\text { HALL / } \\
\text { SCHEINER } \\
(2006)\end{array}$ & $\begin{array}{l}\text { CLAMER et } \\
\text { al. }(2006)\end{array}$ & $\begin{array}{l}\text { BusCHA / } \\
\text { SZITA } \\
(2010)\end{array}$ & $\begin{array}{l}\text { BuSCHA / } \\
\text { SZITA } \\
(2011)\end{array}$ & $\begin{array}{l}\text { BusCHA / } \\
\text { SzITA } \\
(2013)\end{array}$ \\
\hline \multicolumn{7}{|c|}{ Semantik des Konjunktivs I } \\
\hline 1. Indirektheitskonjunktiv & $\begin{array}{l}\text { S. } 122, \\
124\end{array}$ & $\begin{array}{l}\text { S. } 119, \\
120\end{array}$ & S. 105 & & S. 90 & S. 77 \\
\hline \multicolumn{7}{|l|}{ 2. Volitiv } \\
\hline $\begin{array}{l}\text { 2a Direkte Wünsche / } \\
\text { Bitten }\end{array}$ & & S. 118 & S. 107 & & & S. 77 \\
\hline $\begin{array}{l}\text { 2b Indirekte Wünsche / } \\
\text { Bitten (Finalsatz) }\end{array}$ & & S. 119 & & & & \\
\hline $\begin{array}{l}\text { 2c Aufforderung (1. / } 3 . \\
\text { Ps. Pl.) }\end{array}$ & & S. 118 & & & & \\
\hline $\begin{array}{l}\text { 2d Aufforderung } \\
\text { (Fachtexte) }\end{array}$ & & S. 119 & S. 108 & & & \\
\hline $\begin{array}{l}\text { 2e Aufforderung (mit } \\
\text { man) }\end{array}$ & & S. 118 & S. 108 & & & \\
\hline $\begin{array}{l}\text { 2f Einräumung / } \\
\text { Zugeständnis }\end{array}$ & & S. 119 & S. 108 & & & S. 76 \\
\hline 3 Autorenplural & & S. 119 & S. 108 & & & \\
\hline
\end{tabular}

\begin{tabular}{lcccccc}
\hline \multicolumn{7}{c}{ Semantik des Konjunktivs II } \\
\hline $\begin{array}{l}\text { 1. Hypothetische und } \\
\text { irreale Bedingungen }\end{array}$ & S. 121 & S. 94 & S. 96 & S. 53 & S.87 & S. 67 \\
$\begin{array}{l}\text { 2. Un- und erfüllbare } \\
\text { S. } 119\end{array}$ & S. 94 & S. 96 & S. 53 & S. 87 & S. 67
\end{tabular}


Geith, V. - Konjunktiv als Mittel der Bedeutungsschattierung

TABELLE 1a: Semantik von Konjunktiv I und II

Wünsche

3. Irreale Vergleiche

S. 111

S. 95

S. 96

S. 89

S. 67

4. Konjunktiv der

S. 111, S. 95

S. 96

S. 51

S.81

S. 67

Höflichkeit

Bsp.

5. KII als Ersatz für KI in

S. 111,

S. 95

S. 96

S. 90

S. 67 der iR 122

Bsp.

6. Modalverben mit

S. 111

S. 95

S. 96

Konjunktiv

7. Irreale Folgen

S. 111

S. 95

S. 96

8. Irreale Einräumung

S. 95

9. Relativsätze nach

S. 95 negierter Feststellung

10. Sätze mit beinahe / fast

S. 95

S. 89

S. 67

11. Sätze mit sonst / andernfalls

S. 95, S. 105

12. Zweifelnde Fragen /

S. 95

Erstaunen

13. Abschließende

S. 95

Feststellung

14. Unsicherheiten /

Redewendungen mit

Verben des Sagens

15. Die Welt, wie sie ist, sich anders vorstellen

16. Unterscheidung

Realität vs. Irrealität

TABELLE 1b: Semantik von Konjunktiv I und II

\begin{tabular}{l|l|l|l|l|l|l}
\hline & $\begin{array}{l}\text { RUG/ } \\
\text { TOMA- } \\
\text { SZEWSKI } \\
(2009)\end{array}$ & $\begin{array}{l}\text { REIMANN } \\
(2000)\end{array}$ & $\begin{array}{l}\text { HERING et } \\
\text { al. (2009) }\end{array}$ & $\begin{array}{l}\text { FAN-DRYCH } \\
(2012)\end{array}$ & $\begin{array}{l}\text { DREYER / } \\
\text { SCHMITT } \\
(2000)\end{array}$ & $\begin{array}{l}\text { DREYER/ } \\
\text { SCHMITT } \\
(2009)\end{array}$ \\
\hline
\end{tabular}

Semantik des Konjunktivs I

\begin{tabular}{llllllll}
\hline 1. Indirektheitskonjunktiv & S. 103, & S. & 78, & S. 140, & S. 176 & S. 257, & S. 278, \\
& S. 109 & Spez. & Spez. & & S. 275 & S. 298
\end{tabular}

2. Volitiv

2a Direkte Wünsche / 
TABELLE 1b: Semantik von Konjunktiv I und II
Bitten
2b Indirekte Wünsche /
Bitten (Finalsatz)
2c Aufforderung (1./3.
Ps. Pl.)
2d Aufforderung
(Fachtexte)
2e Aufforderung (mit
man)
2f Einräumung /
Zugeständnis
3 Fachtexte

\section{Semantik des Konjunktivs II}

1. Hypothetische und
S. 55

S. 66

S. 134

S. 170

S. 263

S. 285

irreale Bedingungen

2. Un- und erfüllbare

S. 55

S. 67

S. 136

S. 171

S. 261

S. 283

Wünsche

3. Irreale Vergleiche

S. 56

S. 67

S. 138

S. 173

S. 266

S. 288

4. Konjunktiv der

S. 53

S. $66, \mathrm{~S}$.

S. 175

S. 269

S. 292

Höflichkeit 67

5. KII als Ersatz für KI in der iR

S. 104

S. 140

S. 176

6. Modalverben und

S. 174

S. 269

S. 292

Konjunktiv

7. Irreale Folgen

S. 136

S. 173

S. 267

S. 290

8. Irreale Einräumung

S. 171

9. Relativsätze nach negierter Feststellung

10. Sätze mit beinahe / fast

11. Sätze mit sonst / andernfalls

12. Zweifelnde Fragen /

S. 55

S. 270

S. 292

Erstaunen

13. Abschließende

$\begin{array}{ll}\text { S. } 269 & \text { S. } 292\end{array}$

Feststellung

14. Unsicherheiten /

S. 56

S. 265

S. 288

Redewendungen mit

Verben des Sagens

15. Die Welt, wie sie ist,

S. 55

sich anders vorstellen

Pandaemonium, São Paulo, v. 19, n. 29, nov.-dez. 2016, p. 53-94 
Geith, V. - Konjunktiv als Mittel der Bedeutungsschattieruns

TABELLE 1b: Semantik von Konjunktiv I und II

16. Unterscheidung

S. 269

S. 292

Realität vs. Irrealität

TABELLE 2: Deutliche, umfassende und allgemeine Abgrenzung von Konjunktiv zu Indikativ

STEIN-

BASSLER

(2008)

HALL /

SCHEINER

(2006)

CLAMER et al. (2006)

BUSCHA /

SZITA (2010)

BusCha /

SZITA (2011)

BUSCHA /

SZITA (2013)

RUG /

TOMASZEWSKI

(2009)

REIMANN

(2000)

HERING et al.

FANDRYCH

DREYER /

SCHMITT

(2000)

DREYER /

SCHMITT (2009) vorhanden: Der Indikativ beschreibt Handlungen oder Zustände, die in der Realität tatsächlich stattfinden bzw. vorhanden / real sind. Der Konjunktiv beschreibt Wünsche, Pläne, Phantasien, Entwürfe etc., die nur in Gedanken existieren, d.h. sie drücken nicht reale Dinge aus; er dient dazu, sich von Gesagtem zu distanzieren (vgl. S. 110).

vorhanden: Der Indikativ stellt einen Sachverhalt als wirklich / tatsächlich dar, während der Konjunktiv etwa Nicht-Wirkliches, Gedachtes, Hypothetisches, Fiktives beschreibt (vgl. S. 95); die beiden stehen in einem Umkehrverhältnis: Aussagen im Konjunktiv bedeuten, dass etwas in Wirklichkeit nicht so der Fall ist, sondern das Gegenteil (vgl. S. 96).

nicht vorhanden

nicht vorhanden: Gegenüberstellung von Sätzen im Indikativ und Konjunktiv bei Bitten und bei Wünschen (vgl. S. 51, S. 54).

nicht vorhanden

vorhanden: Der Indikativ wird als Normalform sprachlicher Äußerungen bezeichnet, der eine Aussage als Wirklichkeit darstellt und im Sprachgebrauch am häufigsten ist (vgl. S.65). Der Konjunktiv II wird hauptsächlich zum Ausdruck von irrealen Sachverhalten oder besonderer Höflichkeit verwendet. Der Konjunktiv I dient in der Regel zur Wiedergabe von Meinungen und Äußerungen in der indirekten Rede (vgl. S. $65)$.

nicht vorhanden: Nur bei der indirekten Rede wird eine Gegenüberstellung vorgenommen (vgl. S. 103).

nicht vorhanden: Nur bei Konditionalsätzen werden indikativische und konjunktivische Sätze gegenübergestellt. Die Sätze im Indikativ bekommen den Zusatz real, während bei den Sätzen im Konjunktiv irreal steht (vgl. S. 66).

nicht vorhanden: Nur bei Konditionalsätzen werden Beispielsätze im Indikativ dem Konjunktiv gegenübergestellt: Indikativ (realer Plan) vs. Konjunktiv (Wunschraum, irrealer Plan) (vgl. S. 134).

nicht vorhanden: Nur bei den Konditionalsätzen wird der Konjunktiv dem Indikativ gegenübergestellt: nicht erfüllbare Bedingung vs. erfüllbare Bedingung (vgl. S. 170).

vorhanden: Der Indikativ beschreibt etwas Wirkliches oder wirklich Geglaubtes, während der Konjunktiv II etwas Irreales darstellt oder der Wiedergabe fremder Meinungen dient (vgl. S. 257).

bedingt: Der Indikativ wird nur sehr kurz definiert als Verbform, die der Wirklichkeit entsprechen. Ihm wird der Konjunktiv gegenüber gestellt. Er wird definiert als Verbformen, die eine Möglichkeit angeben (vgl. S. 287). Darüber hinaus gibt es keine weitere Differenzierung. 
Geith, V. - Konjunktiv als Mittel der Bedeutungsschattierung

TABELLE 3: Konjunktiv als Mittel der Modalität

\begin{tabular}{|c|c|}
\hline $\begin{array}{l}\text { STEIN-BASSLER } \\
(2008)\end{array}$ & nicht vorhanden \\
\hline $\begin{array}{l}\text { HALL / SCHEINER } \\
(2006)\end{array}$ & nicht vorhanden \\
\hline $\begin{array}{l}\text { CLAMER et al. } \\
(2006)\end{array}$ & nicht vorhanden \\
\hline $\begin{array}{l}\text { BUSCHA / SZITA } \\
(2010)\end{array}$ & nicht vorhanden \\
\hline $\begin{array}{l}\text { BUSCHA / SZITA } \\
(2011)\end{array}$ & nicht vorhanden \\
\hline $\begin{array}{l}\text { BUSCHA / SZITA } \\
(2013)\end{array}$ & $\begin{array}{l}\text { bedingt vorhanden: Indikativ, Imperativ und Konjunktiv werden als Aussageweisen } \\
\text { des Verbes definiert (S. 65). Aber es wird keine weitere Erklärung gegeben, was } \\
\text { unter Aussageweise zu verstehen ist. }\end{array}$ \\
\hline $\begin{array}{l}\text { RUG / } \\
\text { TOMASZEWSKI } \\
(2009)\end{array}$ & nicht vorhanden \\
\hline REIMANN (2000) & nicht vorhanden \\
\hline $\begin{array}{l}\text { HERING et al. } \\
(2009)\end{array}$ & nicht vorhanden \\
\hline $\begin{array}{l}\text { FANDRYCH } \\
(2012)\end{array}$ & $\begin{array}{l}\text { nicht vorhanden: Es gibt zwar ein extra Kapitel über Modalität (vgl. S. 158-175). In } \\
\text { diesem werden Modalverben als Ausdruck von Bedingungen des Handels präsentiert. } \\
\text { Der Konjunktiv wird auch in diesem Kapitel aufgelistet, es wird aber nicht erklärt, } \\
\text { welche Konsequenzen sich daraus ergeben. }\end{array}$ \\
\hline $\begin{array}{l}\text { DREYER / } \\
\text { SCHMITT (2000) }\end{array}$ & $\begin{array}{l}\text { vorhanden: Der Modus wird als Aussageweise definiert und beschrieben, dass mit } \\
\text { dem Modus Indikativ eine Aussage als wirklich oder wirklich geglaubt hingestellt } \\
\text { werden kann. Der Konjunktiv wird als eine andere Aussageweise, ein anderer } \\
\text { Modus, präsentiert, mit dem man die Rede einer anderen Person wiedergibt oder } \\
\text { etwas Irreales, nicht Wirkliches darstellt (vgl. S. 257). }\end{array}$ \\
\hline $\begin{array}{l}\text { DREYER / } \\
\text { SCHMITT (2009) }\end{array}$ & nicht vorhanden \\
\hline
\end{tabular}

\section{Literaturverzeichnis}

Althaus, Hans Peter et al. (Hg.). Lexikon der Germanistischen Linguistik. 2. vollständig neu bearbeitete und erweiterte Auflage. Max Niemeyer Verlag, 1980.

BusCHA, Anne;SZITA, Szilvia. A Grammatik. Übungsgrammatik Deutsch als Fremdsprache. Sprachniveau A1 - A2. Leipzig: Schubert-Verlag, 2010.

Buscha, Anne; SZITA, Szilvia. B Grammatik. Übungsgrammatik Deutsch als Fremdsprache. Sprachniveau B1 - B2. Leipzig: Schubert-Verlag, 2011.

BuschA, Anne; SzITA, Szilvia. C Grammatik. Übungsgrammatik Deutsch als Fremdsprache. Sprachniveau C1-C2. Leipzig: Schubert-Verlag, 2013.

Bubmann, Hadumod (Hg.). Lexikon der Sprachwissenschaft. Dritte, aktualisierte und erweiterte Auflage Stuttgart: Alfred Körner Verlag, 2002. 
Geith, V. - Konjunktiv als Mittel der Bedeutungsschattierung

CLAMER, Friedrich et. al. Übungsgrammatik für die Mittelstufe. Regeln. Listen. Übungen. Erweiterte Fassung. Zweite, korrigierte Auflage. Bonn: Liebaug-Dartmann, 2006.

CONRAD, Rudi (Hg.). Lexikon sprachwissenschaftlicher Termini. Leipzig: Bibliographisches Institut, 1985.

DREYER, Heicke; SCHMITT, Richard. Lehr- und Übungsgrammatik der deutschen Grammatik. Die neue Gelbe. Ismaning: Hueber Verlag, 2000.

DREYER, Heicke; SCHMITT, Richard. Lehr- und Übungsgrammatik der deutschen Grammatik. Die Gelbe aktuell. Ismaning: Hueber Verlag, 2009.

DuDEN. Die Grammatik. Unentbehrlich für richtiges Deutsch. 7. völlig neu erarbeitete und erweiterte Auflage. Duden Band IV. Mannheim / Leipzig / Wien / Zürich: Dudenverlag, 2006.

DUDEN. Die Grammatik. Unentbehrlich für richtiges Deutsch. 8. überarbeitete Auflage. Duden Band IV. Mannheim,Leipzig,Wien, Zürich: Dudenverlag, 2009.

ENGEL, Ulrich. Deutsche Grammatik. 3. korrigierte Auflage. Heidelberg: Julius Groos Verlag, 1996.

ENGEL, Ulrich. Deutsche Grammatik. Neubearbeitung. Gerstenhofen: Iudicium, 2004.

FANDRYCH, Christian (Hg.). Klipp und Klar. Übungsgrammatik Mittelstufe B2 / C1. Deutsch als Fremdsprache. Stuttgart: Klett Verlag, 2012.

GLÜCK, Helmut (Hg.). Metzler Lexikon Sprache. 3. neuüberarbeitete Auflage mit 70 Abbildungen, davon 17 Karten. Stuttgart: Verlag J.B. Metzler, 2000.

GÖTZE, Lutz et al. Lehrschwierigkeiten im Fach ,Deutsch als Fremdsprache : Abschlussbericht. München: Goethe-Institut, Arbeitsstelle für wissenschaftliche Didaktik, 1979.

GRIESBACH, Heinz. Neue deutsche Grammatik. 5. Auflage. Berlin / München: Langenscheidt, 1990

HALL, Karin; SCHEINER, Barbara. Übungsgrammatik für Fortgeschrittene. Deutsch als Fremdsprache. 4. unveränderte Auflage. München Hueber Verlag, 2006.

HEIDOLPH, Karl et al. Grundzüge einer deutschen Grammatik. Berlin: Akademie Verlag, 1981.

Helbig, Gerhard;; BuschA, Joachim. Deutsche Grammatik. Ein Handbuch für den Ausländerunterricht. 5. Auflage. Berlin / München: Langenscheidt, 2005.

HENTSCHEL, Elke; WEYDT, Harald: Handbuch der deutschen Grammatik. 3. völlig neu bearbeitete Auflage. Berlin, New York: Walter de Gruyter, 2003.

HERING et al., Axel et al. Übungsgrammatik für die Mittelstufe. Ismaning: Hueber Verlag, 2009.

HOMBERGER, Dietrich. Sachwörterbuch zur Sprachwissenschaft. Stuttgart: Reclam, 2000

JUNG, Walter. Grammatik der deutschen Sprache. 8. durchgesehene Auflage. Leipzig: Bibliographisches Institut, 1984.

ReIMANN, Monika. Grundstufen-Grammatik für Deutsch als Fremdsprache. Erklärungen und Übungen. 3. Auflage. Ismaning: Hueber Verlag, 2000.

RUG, Wolfgang; TOMASZEWSKI, Andreas. Grammatik mit Sinn und Verstand. Übungsgrammatik Mittel- und Oberstufe. Stuttgart: Klett Verlag, 2009.

SOMMERFELDT, Karl-Ernst; STARKE, Günter. Einführung in die Grammatik der deutschen Gegenwartssprache. 3., neu bearbeitete Auflage unter Mitwirkung von Werner Hackel. Tübingen: Max Niemeyer Verlag, 1998.

STEIN-BASSLER, Dorothea. Lernergrammatik zur Studienvorbereitung. Meckenheim: Verlag Liebaug-Dartmann, 2008.

ZIFOnUn, Gisela et al. Grammatik der deutschen Sprache. Band III. Schriften des Instituts für deutsche Sprache. Band 7.3. Berlin, New York: Walter de Gruyter, 1997.

Recebido em 06/06/2016 Aceito em 29/08/2016 